\title{
Ground-state properties of the symmetric single-impurity Anderson model on a ring from density-matrix renormalization group, Hartree-Fock, and Gutzwiller theory
}

\author{
Gergely Barcza, ${ }^{1}$ Florian Gebhard, ${ }^{2, *}$ Thorben Linneweber, ${ }^{2}$ and Örs Legeza ${ }^{1}$ \\ ${ }^{1}$ Strongly Correlated Systems Lendület Research Group, Institute for Solid State Physics and Optics, MTA Wigner Research Centre for \\ Physics, P.O. Box 49, H-1525 Budapest, Hungary \\ ${ }^{2}$ Fachbereich Physik, Philipps-Universität Marburg, D-35032 Marburg, Germany
}

(Received 5 June 2018; revised manuscript received 25 March 2019; published 19 April 2019)

\begin{abstract}
We analyze the ground-state energy, magnetization, magnetic susceptibility, and Kondo screening cloud of the symmetric single-impurity Anderson model (SIAM) that is characterized by the bandwidth $W$, the impurity interaction strength $U$, and the local hybridization $V$. We compare Gutzwiller variational and magnetic HartreeFock results in the thermodynamic limit with numerically exact data from the density-matrix renormalization group (DMRG) method on large rings. To improve the DMRG performance, we use a canonical transformation to map the SIAM onto a chain with half the system size and open boundary conditions. We compare to Bethe ansatz results for the ground-state energy, magnetization, and spin susceptibility that become exact in the wide-band limit. Our detailed comparison shows that the field-theoretical description is applicable to the SIAM on a ring for a broad parameter range. Hartree-Fock theory gives an excellent ground-state energy and local moment for intermediate and strong interactions. However, it lacks spin fluctuations and thus cannot screen the impurity spin. The Gutzwiller variational energy bound becomes very poor for large interactions because it does not describe properly the charge fluctuations. Nevertheless, the Gutzwiller approach provides a qualitatively correct description of the zero-field susceptibility and the Kondo screening cloud. The DMRG provides excellent data for the ground-state energy and the magnetization for finite external fields. At strong interactions, finite-size effects make it extremely difficult to recover the exponentially large zero-field susceptibility and the mesoscopically large Kondo screening cloud.
\end{abstract}

DOI: 10.1103/PhysRevB.99.165130

\section{INTRODUCTION}

The single-impurity Anderson model (SIAM) decribes an impurity in a metallic host in which electrons interact locally $[1,2]$. It poses one of the best studied and understood fundamental many-body problems; for a review, see Ref. [3]. Therefore, it still serves as a benchmark test for the development of advanced analytical many-body techniques, e.g., the functional renormalization group technique [4-6]. For the symmetric SIAM, the low-energy physics is similar to that of the single-impurity $s$ - $d$ or Kondo model $[7,8]$ in which an impurity spin couples to the host electrons' spin degrees of freedom: at zero temperature, the impurity local moment is screened by the host electrons, which gives rise to a narrow Abrikosov-Suhl or Kondo resonance in the impurity spectral function at the Fermi level [3]. The resonance can be resolved using the numerical renormalization group (NRG) technique; for a review, see Ref. [9]. At higher energies, Hubbard satellites appear in the impurity spectral function that describe the local charge fluctuations. Both the Kondo resonance and the Hubbard satellite are accessible from the analytic local-moment approach [10-12].

More recently, the real-space features of the screening were studied for the Kondo model using NRG [13-15] and the

*florian.gebhard@physik.uni-marburg.de analytical coherent-state expansion [16]. For the noninteracting SIAM (resonant-level model) in the wide-band limit, the screening cloud was analyzed analytically [17] and the magnetic properties of the interacting SIAM were studied numerically using the density-matrix renormalization group (DMRG) method [18]. The various methods show that the screening cloud extends very far into the host metal. In the Kondo regime, an algebraic decay sets in only beyond a characteristic (Kondo) length scale that is proportional to the inverse of the Kondo temperature.

Less attention was dedicated to ground-state properties of the SIAM and Kondo models because they are solvable by the Bethe ansatz [19-22]. Therefore, important quantities such as the ground-state energy, magnetization, and magnetic susceptibility at zero field are known explicitly. The Bethe ansatz is based on the wide-band limit, $W \rightarrow \infty$, so that the dispersion relation of the host electrons can be linearized around the Fermi energy. However, the implicit assumption that the Hubbard interaction $U$ is small compared to the bandwidth, $U \ll W$, impedes a comparison with methods that treat the SIAM on a lattice such as the DMRG method and the Hartree-Fock [1] and Gutzwiller wave functions [23,24].

As one of the best studied many-body problems, the SIAM is particularly suitable to test existing and conceivable future many-body methods. Since these are often customized for the treatment of lattice Hamiltonians, it is one of the purposes of this work to provide tangible results for a ring geometry; 
for other recent numerical treatments of finite structures, see Refs. [25,26]. Given the high accuracy of the DMRG data for large system sizes, an extrapolation of most ground-state properties to the thermodynamic limit is unproblematic. As we shall see, the wide-band limit remains applicable for fairly large interaction strengths even for a substantial hybridization that justifies the application of the wide-band limit even for sizable Coulomb parameters.

In this work, we use the DMRG to calculate numerically exactly the ground-state energy, the local magnetic moment, the zero-field susceptibility, and the screening cloud of the single-impurity Anderson model on large rings. The Gutzwiller and Hartree-Fock approaches provide complementary insights. The Hartree-Fock variational estimate of the ground-state energy is very satisfactory for moderate to large Hubbard interactions whereas the Gutzwiller estimate is acceptable only for small $U$. On the other hand, the Gutzwiller approach provides a qualitatively correct description of the magnetic properties whereas Hartree-Fock theory fails to screen the impurity spin even at infinitely large distances. Since the Gutzwiller approach is heavily based on the exact results for the noninteracting SIAM, we compile the results for the resonant-level model in the Supplemental Material [27].

Our work is structured as follows. In Sec. II, we introduce the one-dimensional SIAM on a ring with local hybridization at particle-hole and spin symmetry. We map the model onto a two-chain problem $[28,29]$ in which the two chains separate in the thermodynamic limit. The reduced model provides the basis for our numerical DMRG investigations. In Sec. III we discuss the ground-state energy, magnetization, and spin correlation function between the impurity and the bath sites for the noninteracting SIAM for small hybridizations. The derivation of the formulas is deferred to the Supplemental Material [27]. In Sec. IV, we evaluate the Gutzwiller variational wave function for the SIAM and determine an analytical variational upper bound for the ground-state energy. Moreover, we calculate the variational magnetization and spin correlation function. In Sec. V we compare our results for the groundstate energy, magnetization, and the spin correlation function with numerically exact DMRG data for large system sizes. We include the results from the Bethe ansatz and a magnetic Hartree-Fock calculation; see the Supplemental Material for their derivation [27]. Short conclusions, Sec. VI, close our presentation.

\section{SYMMETRIC SINGLE-IMPURITY ANDERSON MODEL ON A RING}

We study the particle-hole and spin symmetric SIAM on a ring [1,2]. For strong interactions, this model maps onto the one-dimensional Kondo impurity model [30].

\section{A. Hamiltonian}

The Hamilton operator for the one-dimensional singleimpurity Anderson model reads $[1,3]$

$$
\begin{aligned}
& \hat{H}=\hat{H}_{0}+\hat{H}_{\text {int }}, \\
& \hat{H}_{\text {int }}=U\left(\hat{n}_{d, \uparrow}-1 / 2\right)\left(\hat{n}_{d, \downarrow}-1 / 2\right),
\end{aligned}
$$

where $\hat{n}_{d, \sigma}=\hat{d}_{\sigma}^{+} \hat{d}_{\sigma}$ counts the number of $\sigma$ electrons on the impurity site $(\sigma=\uparrow, \downarrow)$. Only the electrons on the impurity site repel each other with strengths $U>0$. The noninteracting Hamiltonian,

$$
\hat{H}_{0}=\hat{T}+\hat{B}+\hat{V}+\hat{P},
$$

describes bath electrons that move between neighboring sites on a ring with $L$ sites,

$$
\hat{T}=-\frac{W}{4} \sum_{n=0, \sigma}^{L-1}\left(\hat{c}_{n, \sigma}^{+} \hat{c}_{n+1, \sigma}+\hat{c}_{n+1, \sigma}^{+} \hat{c}_{n, \sigma}\right),
$$

where the bandwidth provides our unit of energy, $W \equiv 1$.

In the presence of an external magnetic field $\mathcal{H}_{\text {bath }}$ we may include the magnetic term

$$
\hat{B}=-B_{\text {bath }} \sum_{n=0}^{L-1}\left(\hat{c}_{n, \uparrow}^{+} \hat{c}_{n, \uparrow}-\hat{c}_{n, \downarrow}^{+} \hat{\iota}_{n, \downarrow}\right) .
$$

Here, we abbreviated $B_{\text {bath }}=g \mu_{\mathrm{B}} \mathcal{H}_{\text {bath }} / 2$, where $\mu_{\mathrm{B}}$ is the Bohr magneton and $g \approx 2$ is the electrons' gyromagnetic factor.

The bath electrons hybridize at the origin, $n=0$, with the impurity electrons with strength $V>0$,

$$
\hat{V}=V \sum_{\sigma}\left(\hat{d}_{\sigma}^{+} \hat{c}_{0, \sigma}+\hat{c}_{0, \sigma}^{+} \hat{d}_{\sigma}\right) .
$$

The system is half filled; i.e., the total number of electrons is $N=L+1$, and we investigate a paramagnetic situation, $N_{\uparrow}=$ $N_{\downarrow}=(L+1) / 2$. Consequently, the number of bath sites $L$ must be odd. From now on we further assume that $(L+3) / 2$ is even.

There can be a local, possibly spin-dependent potential,

$$
\hat{P}=-\sum_{\sigma} E_{d, \sigma}\left(\hat{n}_{d, \sigma}-1 / 2\right) .
$$

In the presence of an external magnetic field $\mathcal{H}_{\text {imp }}$ at the impurity we have $E_{d, \uparrow}=\left(g \mu_{\mathrm{B}} / 2\right) \mathcal{H}_{\mathrm{imp}}=-E_{d, \downarrow}$. In the magnetic Hartree-Fock approach, we have $E_{d, \uparrow}=U m=-E_{d, \downarrow}$, where the value of the $\hat{S}_{z}$ at the impurity, $m=\left\langle\hat{n}_{d, \uparrow}-\hat{n}_{d, \downarrow}\right\rangle / 2$, has to be determined self-consistently [27].

\section{B. Particle-hole symmetry}

To analyze particle-hole symmetry in the SIAM, we set $E_{d, \sigma}=0$ and $\mathcal{H}_{\text {bath }}=0$ in the rest of this section; i.e., we have no magnetic symmetry breaking. Particle-hole symmetry for the SIAM was studied previously, e.g., in Ref. [31].

The ring geometry renders the analysis of particle-hole symmetry more cumbersome than the choice of open boundary conditions. Since boundary conditions play no role in the thermodynamic limit as investigated in Secs. III-V, the material presented in this section is included for completeness rather than necessity.

\section{Wave numbers and particle-hole boundary conditions}

For a ring, the kinetic energy is diagonal in momentum space,

$$
\hat{T}=\sum_{k, \sigma} \epsilon(k) \hat{c}_{k, \sigma}^{+} \hat{c}_{k, \sigma}
$$


where

$$
\hat{c}_{n, \sigma}=\sqrt{\frac{1}{L}} \sum_{k} e^{i k n} \hat{c}_{k, \sigma}, \quad \hat{c}_{k, \sigma}=\sqrt{\frac{1}{L}} \sum_{n=0}^{L-1} e^{-i k n} \hat{c}_{n, \sigma},
$$

and the dispersion relation is given by

$$
\epsilon(k)=-\cos (k) / 2 .
$$

In one dimension $Q=\pi$ is half a reciprocal lattice vector, $\epsilon(k+2 Q)=\epsilon(k)$. For particle-hole symmetry we must demand that

$$
\epsilon(\pi-k)=-\epsilon(k)
$$

for all accessible $|k| \leqslant \pi$. In particular, this equation implies that with $k$, also $\pi-k$ is an accessible $k$ value. This is not difficult to fulfill for even $L$ but poses a problem for odd $L$.

Let

$$
k_{m}=\frac{2 \pi}{L} m+\varphi, m=-(L-1) / 2, \ldots,(L-1) / 2,
$$

where $0 \leqslant \varphi<2 \pi / L$ and $k_{m}$ are defined modulo $2 \pi$. Then, the set of $k$ values must also be given by

$$
\begin{aligned}
k_{m}^{\prime} & =\pi-\frac{2 \pi}{L} m-\varphi=\frac{2 \pi}{L}\left(\frac{L-1}{2}+\frac{1}{2}-m\right)+\varphi-2 \varphi \\
& =\frac{2 \pi}{L}\left(\frac{L-1}{2}-m\right)+\varphi+\frac{\pi-2 \varphi L}{L} .
\end{aligned}
$$

Using the definition of the accessible $k$ values, we see that we must set

$$
\varphi= \pm \pi /(2 L)
$$

to make the sets $\{k\}$ and $\left\{k^{\prime}\right\}$ identical. Particle-hole symmetry for odd $L$ destroys inversion symmetry because the energy levels $\epsilon(k)$ are not degenerate; i.e., if $k$ is an allowed value, $k^{\prime}=-k$ is not accessible.

The accessible $k$ values belong to the boundary conditions

$$
e^{i k_{m} L}=e^{i \pi / 2}=i
$$

i.e., they are neither periodic nor antiperiodic. We call these boundary conditions particle-hole periodic. They imply $\hat{c}_{L}=$ $i \hat{c}_{0}\left(\hat{c}_{L}^{+}=-i \hat{c}_{0}^{+}\right)$in position space so that we may write for the kinetic energy in Eq. (2)

$$
\begin{aligned}
\hat{T}= & -\frac{1}{4} \sum_{n=0, \sigma}^{L-2}\left(\hat{c}_{n, \sigma}^{+} \hat{c}_{n+1, \sigma}+\hat{c}_{n+1, \sigma}^{+} \hat{c}_{n, \sigma}\right) \\
& -\frac{1}{4}\left(i \hat{c}_{L-1, \sigma}^{+} \hat{c}_{0, \sigma}-i \hat{c}_{0, \sigma}^{+} \hat{c}_{L-1, \sigma}\right) .
\end{aligned}
$$

Equation (15) shows that the kinetic energy is indeed invariant under the particle-hole transformation

$$
\mathrm{ph}: \quad \hat{c}_{n, \sigma} \mapsto(-1)^{n} \hat{c}_{n, \sigma}^{+} \quad \text { for } n=0,1, \ldots, L-1,
$$

because either $n$ or $n+1$ is even when the other is odd, and the origin and $L-1$ are both even numbers for odd $L$.

\section{Model properties at particle-hole symmetry}

For the one-dimensional model (2) with particle-hole boundary conditions and $E_{d, \sigma}=0$, we define the particle-hole transformation

$$
\begin{aligned}
\mathrm{ph}: \hat{c}_{n, \sigma} & \mapsto(-1)^{n} \hat{c}_{n, \sigma}^{+} \quad \text { for } n=0,1, \ldots, L-1, \\
\hat{d}_{\sigma} & \mapsto(-1) \hat{d}_{\sigma}^{+} .
\end{aligned}
$$

It is readily seen that the transformation leaves the Hamiltonian $\hat{H}$ invariant. The particle-number operators transform according to

$$
\hat{n}_{d, \sigma} \mapsto 1-\hat{n}_{d, \sigma}, \quad \hat{c}_{n, \sigma}^{+} \hat{c}_{n, \sigma} \mapsto 1-\hat{c}_{n, \sigma}^{+} \hat{c}_{n, \sigma},
$$

so that the $N$-particle sector maps onto the sector with $2(L+$ 1) $-N$ particles. At half band filling, $N=L+1$, the normalized ground state maps onto itself, $\left|\Psi_{0}\right\rangle \mapsto\left|\Psi_{0}\right\rangle$, up to a global phase. Therefore, particle-hole symmetry guarantees

$$
\left\langle\Psi_{0}\left|\hat{n}_{d, \sigma}\right| \Psi_{0}\right\rangle=1 / 2
$$

for all interaction strengths $U$ and hybridizations $V$. Moreover, we obtain

$$
\begin{aligned}
\left\langle\Psi_{0}\left|\hat{c}_{n, \sigma}^{+} \hat{d}_{\sigma}\right| \Psi_{0}\right\rangle & =(-1)^{n}\left\langle\Psi_{0}\left|\hat{d}_{\sigma}^{+} \hat{c}_{n, \sigma}\right| \Psi_{0}\right\rangle \\
& =(-1)^{n}\left\langle\Psi_{0}\left|\hat{c}_{n, \sigma}^{+} \hat{d}_{\sigma}\right| \Psi_{0}\right\rangle^{*}
\end{aligned}
$$

for the hybridization matrix element between impurity and bath electrons at site $n$. Therefore, the matrix elements are alternately real or purely imaginary. In momentum space, Eq. (20) reads

$$
M(k) \equiv\left\langle\Psi_{0}\left|\hat{c}_{k, \sigma}^{+} \hat{d}_{\sigma}\right| \Psi_{0}\right\rangle=\left\langle\Psi_{0}\left|\hat{c}_{\pi-k, \sigma}^{+} \hat{d}_{\sigma}\right| \Psi_{0}\right\rangle^{*} .
$$

Since the wave numbers $k$ enter the single-impurity Anderson model only via the dispersion relation, $M(k) \equiv M(\epsilon(k))$, Eq. (21) implies

$$
\operatorname{Re} M(-\epsilon)=\operatorname{Re} M(\epsilon), \quad \operatorname{Im} M(-\epsilon)=-\operatorname{Im} M(\epsilon),
$$

because $\epsilon(\pi-k)=-\epsilon(k)$. We shall use this relation in Sec. III.

\section{Phase shifts and periodic boundary conditions}

Instead of using particle-hole periodic boundary conditions, we may distribute the phase shift $\Phi= \pm \pi / 2$ evenly and use periodic boundary conditions. We rewrite

$$
\hat{c}_{n, \sigma}=\exp (i \varphi n) \hat{b}_{n, \sigma}
$$

for $n=0,1, \ldots, L-1$. Then,

$$
\hat{T}=-\frac{1}{4} \sum_{n=0, \sigma}^{L-1}\left(e^{i \varphi} \hat{b}_{n, \sigma}^{+} \hat{b}_{n+1, \sigma}+e^{-i \varphi} \hat{b}_{n+1, \sigma}^{+} \hat{b}_{n, \sigma}\right),
$$

where $\hat{b}_{L, \sigma}=\hat{b}_{0, \sigma}$; i.e., the $b$ electrons obey periodic boundary conditions.

When we Fourier transform into momentum space, we use the wave numbers

$$
\tilde{k}_{m}=\frac{2 \pi}{L} m, \quad m=0,1, \ldots, L-1 .
$$

The kinetic energy becomes

$$
\hat{T}=\sum_{\tilde{k}, \sigma}[-2 t \cos (\tilde{k}+\varphi)] \hat{b}_{\tilde{k}, \sigma}^{+} \hat{b}_{\tilde{k}, \sigma} .
$$

Therefore, the dispersion relation and the set of accessible $k$ values are still given by Eqs. (9) and (11). 
The kinetic energy operator (24) is particle-hole symmetric under the transformation

$$
\text { ph : } \quad \hat{b}_{n, \sigma} \mapsto(-1)^{n} e^{-2 i \varphi n} \hat{b}_{n, \sigma}^{+} \quad \text { for } \quad n=0,1, \ldots, L-1 .
$$

This is readily seen for all electron transfers between sites $n$ and $(n+1)$ for $n=0,1, \ldots,(L-2)$, where the value of $\varphi$ is actually irrelevant. For the electron transfer between the last and first site, however, we find

$$
\begin{aligned}
& e^{i \varphi} \hat{b}_{L-1, \sigma}^{+} \hat{b}_{0, \sigma}+e^{-i \varphi} \hat{b}_{0, \sigma}^{+} \hat{b}_{L-1, \sigma} \mapsto e^{i \varphi} e^{2 i \varphi(L-1)} \hat{b}_{L-1, \sigma} \hat{b}_{0, \sigma}^{+} \\
& \quad+e^{-i \varphi} \hat{b}_{0, \sigma} e^{-2 i \varphi(L-1)} \hat{b}_{L-1, \sigma}^{+}
\end{aligned}
$$

because both the origin and the last site are even. For the transformed term to become equivalent to the original term, we must impose

$$
e^{2 i \varphi L}=-1,
$$

which again gives $\varphi= \pm \pi /(2 L)$ as in Eq. (13).

\section{Mapping onto a chain problem \\ 1. Canonical transformation}

For $n=1,2, \ldots,(L-1) / 2$ we perform the canonical transformation $[28,29]$

$$
\begin{aligned}
\hat{C}_{n, \sigma} & =\sqrt{\frac{1}{2}}\left(e^{i n \varphi} \hat{b}_{n, \sigma}+e^{-i n \varphi} \hat{b}_{L-n, \sigma}\right), \\
\hat{S}_{n, \sigma} & =\sqrt{\frac{1}{2}}\left(e^{i n \varphi} \hat{b}_{n, \sigma}-e^{-i n \varphi} \hat{b}_{L-n, \sigma}\right)
\end{aligned}
$$

with the inverse transformation

$$
\begin{aligned}
\hat{b}_{n, \sigma} & =\sqrt{\frac{1}{2}} e^{-i n \varphi}\left(\hat{C}_{n, \sigma}+\hat{S}_{n, \sigma}\right), \\
\hat{b}_{L-n, \sigma} & =\sqrt{\frac{1}{2}} e^{i n \varphi}\left(\hat{C}_{n, \sigma}-\hat{S}_{n, \sigma}\right) .
\end{aligned}
$$

The kinetic energy becomes

$$
\begin{aligned}
-4 \hat{T}= & \sum_{\sigma}\left(\hat{B}_{1, \sigma}+\hat{B}_{2, \sigma}\right)+\sum_{n=1, \sigma}^{(L-3) / 2}\left(\hat{C}_{n, \sigma}^{+} \hat{C}_{n+1, \sigma}+\hat{C}_{n+1, \sigma}^{+} \hat{C}_{n, \sigma}\right) \\
& +\sum_{n=1, \sigma}^{(L-3) / 2}\left(\hat{S}_{n, \sigma}^{+} \hat{S}_{n+1, \sigma}+\hat{S}_{n+1, \sigma}^{+} \hat{S}_{n, \sigma}\right)
\end{aligned}
$$

where the boundary term at the left chain end reads

$$
\hat{B}_{1, \sigma}=\sqrt{2}\left(\hat{c}_{0, \sigma}^{+} \hat{C}_{1, \sigma}+\hat{C}_{1, \sigma}^{+} \hat{c}_{0, \sigma}\right) .
$$

In contrast to open boundary conditions or boundary conditions that violate particle-hole symmetry, the connection term between the $C$ electrons and $S$ electrons is finite. The term at $n=(L-1) / 2$ is given by

$$
\hat{B}_{2, \sigma}=i\left(\hat{S}_{(L-1) / 2, \sigma}^{+} \hat{C}_{(L-1) / 2, \sigma}-\hat{C}_{(L-1) / 2, \sigma}^{+} \hat{S}_{(L-1) / 2, \sigma}\right) .
$$

The ring and two-chain geometries are shown in Fig. 1.

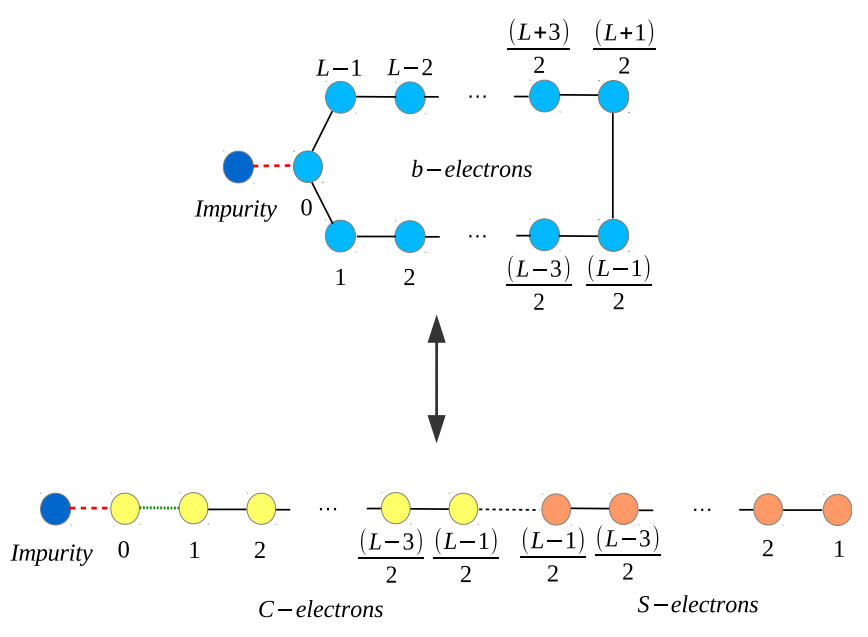

FIG. 1. SIAM in ring geometry, Eq. (1), with the kinetic energy from Eq. (24), and in two-chain geometry with the kinetic energy from Eq. (32). Bonds with the same color have the same electron transfer amplitudes. The dotted bond between the $C$-electron and $S$ electron chains has a complex hopping amplitude.

Note that the particle-hole transformation for the kinetic energy in the two-chain formulation is nontrivial,

$$
\begin{aligned}
\mathrm{ph}:(-1)^{n} e^{-i n \varphi} \hat{C}_{n, \sigma} & \mapsto \cos (\varphi n) \hat{C}_{n, \sigma}^{+}-i \sin (\varphi n) \hat{S}_{n, \sigma}^{+} \\
(-1)^{n} e^{-i n \varphi} \hat{S}_{n, \sigma} & \mapsto \cos (\varphi n) \hat{S}_{n, \sigma}^{+}-i \sin (\varphi n) \hat{C}_{n, \sigma}^{+}
\end{aligned}
$$

for $n=1, \ldots, L-1$, and $\hat{c}_{0, \sigma} \mapsto \hat{c}_{0, \sigma}^{+}$as before.

For comparison, we give in the Appendix the standard derivation of the chain geometry from the ring geometry via the Lanczos procedure [25]. The chains of $C$ electrons and $S$ electrons do not decouple because particle-hole symmetry for odd chain lengths $L$ is not compatible with inversion symmetry. Apparently, it is not advantageous numerically to investigate a ring geometry at particle-hole symmetry. It is more favorable to start from an inversion-symmetric chain where the $C$-electron and $S$-electron chains decouple. In the following we shall investigate the consequences of an $\mathrm{ad} \mathrm{hoc}$ decoupling of the two chains. Note that this does not influence the results in the thermodynamic limit where boundary conditions become irrelevant.

\section{Chain separation}

For large rings, the interchain coupling is small for two reasons. First, as seen from Eq. (34), the chains for the $C$ electrons and $S$ electrons are coupled at a single site only, namely, at the chain center $n=(L-1) / 2$. Second, in the SIAM the interesting physics happens at and around the origin, i.e., at the left boundary of the $C$-electron chain. Because of their large separation, we can expect that the right half of the chain has little effect on the physics at the left boundary.

The chain-separated SIAM reads

$$
\hat{H}=\hat{H}^{C}+\hat{T}^{S} .
$$


The undisturbed chain of antisymmetric standing waves of length $(L-1) / 2$ is described by

$$
\hat{T}^{S}=-\frac{1}{4} \sum_{n=1, \sigma}^{(L-3) / 2}\left(\hat{S}_{n, \sigma}^{+} \hat{S}_{n+1, \sigma}+\hat{S}_{n+1, \sigma}^{+} \hat{S}_{n, \sigma}\right) .
$$

The electrons on the chain of symmetric standing waves of length $(L+1) / 2$ couple to the impurity at the origin,

$$
\begin{aligned}
\hat{H}^{C}= & \hat{H}_{0}^{C}+U\left(\hat{n}_{d, \uparrow}-\frac{1}{2}\right)\left(\hat{n}_{d, \downarrow}-\frac{1}{2}\right), \\
\hat{H}_{0}^{C}= & \hat{T}^{C}+\hat{V}, \\
\hat{T}^{C}= & -\frac{\sqrt{2}}{4} \sum_{\sigma}\left(\hat{C}_{0, \sigma}^{+} \hat{C}_{1, \sigma}+\hat{C}_{1, \sigma}^{+} \hat{C}_{0, \sigma}\right) \\
& -\frac{1}{4} \sum_{n=1, \sigma}^{(L-3) / 2}\left(\hat{C}_{n, \sigma}^{+} \hat{C}_{n+1, \sigma}+\hat{C}_{n+1, \sigma}^{+} \hat{C}_{n, \sigma}\right), \\
\hat{V}= & V \sum_{\sigma}\left(\hat{d}_{\sigma}^{+} \hat{C}_{0, \sigma}+\hat{C}_{0, \sigma}^{+} \hat{d}_{\sigma}\right),
\end{aligned}
$$

where we identified $\hat{c}_{0, \sigma} \equiv \hat{C}_{0, \sigma}$ to keep the notation consistent.

When we ignore the chain coupling term $\hat{B}_{2, \sigma}$, we can factorize the ground state into the contributions from the chains $C$ and $S$,

$$
\left|\Psi_{0}\right\rangle=\left|\Psi_{0}^{C}\right\rangle\left|\Psi_{0}^{S}\right\rangle
$$

where the upper index refers to the two commuting parts of the Hamiltonians for the $C$ electrons and $S$ electrons, and $\left|\Psi_{0}^{C, S}\right\rangle$ are normalized to unity.

The mapping is advantageous for the DMRG treatment because we do not have to treat a ring geometry of $L$ sites with periodic boundary conditions but a chain with $(L+1) / 2+1$ sites, where open boundary conditions apply. The $C$-electron chain has only about half as many sites as the ring, which essentially doubles the system sizes that can be treated numerically for the ring geometry.

Note, however, that $\hat{H}^{C}$ does not obey particle-hole symmetry for finite $L$ but only in the thermodynamic limit. Deviations from particle-hole symmetry can be monitored by investigating the site occupancy of the impurity. Deviations from the exact value of one-half, see Eq. (19), can be used to quantify the violation of particle-hole symmetry; see Sec. V.

In the numerical renormalization group approach, the SIAM is directly considered in energy space. After an appropriate discretization, the resulting Wilson chain is treated numerically [9]. In our approach, we map the Hamiltonian on finite rings to a chain while keeping particle-hole symmetry and the bandwidth finite. The use of a Hamiltonian on a ring geometry permits the direct application, comparison, and assessment of lattice-based variational methods such as Hartree-Fock, Gutzwiller, and DMRG, as done in this work. Our results also permit us to assess the quality of other present and conceivable future many-body methods for lattice Hamiltonians.

\section{Spin correlation function}

In this work we visualize the Kondo screening cloud for the single-impurity Anderson model. To this end, we calculate the spin correlation function between the impurity and bath sites.

\section{Definition and general properties}

Due to the spin-rotational invariance of the model it is sufficient to study the spin correlation function along the spin quantization axis. The local correlation function is defined by

$$
\begin{aligned}
C_{d d}^{S} & =\left\langle\Psi_{0}\left|\hat{S}_{d}^{z} \hat{S}_{d}^{z}\right| \Psi_{0}\right\rangle=\frac{1}{4}\left\langle\Psi_{0}\left|\left(\hat{n}_{\uparrow}^{d}-\hat{n}_{\downarrow}^{d}\right)^{2}\right| \Psi_{0}\right\rangle \\
& =\frac{1}{4}-\frac{1}{2}\left\langle\Psi_{0}\left|\hat{n}_{\uparrow}^{d} \hat{n}_{\downarrow}^{d}\right| \Psi_{0}\right\rangle,
\end{aligned}
$$

where we used particle-hole symmetry (19) in the last step. The value for the on-site spin correlation interpolates between the itinerant limit, $C_{d d}(U=0)=1 / 8$, and the atomic limit, $C_{d d}(W=0)=1 / 4$.

The correlation function between the impurity site and the bath site $r$ is defined by

$$
\begin{aligned}
C_{d c}^{S}(r) & =\left\langle\Psi_{0}\left|\hat{S}_{d}^{z} \hat{S}_{r, c}^{z}\right| \Psi_{0}\right\rangle \\
& =\frac{1}{4}\left\langle\Psi_{0}\left|\left(\hat{n}_{\uparrow}^{d}-\hat{n}_{\downarrow}^{d}\right)\left(\hat{c}_{r, \uparrow}^{+} \hat{c}_{r, \uparrow}-\hat{c}_{r, \downarrow}^{+} \hat{c}_{r, \downarrow}\right)\right| \Psi_{0}\right\rangle .
\end{aligned}
$$

Due to inversion symmetry we have

$$
C_{d c}^{S}(L-r)=C_{d c}^{S}(r)
$$

for $1 \leqslant r \leqslant(L-1) / 2$.

To visualize the screening of the impurity spin, we define $\mathcal{S}(0)=C_{d d}^{S}+C_{d c}^{S}(0)$ and, for $R \geqslant 1$,

$$
\mathcal{S}(R)=C_{d d}^{S}+C_{d c}^{S}(0)+\sum_{r=1}^{R}\left[C_{d c}^{S}(r)+C_{d c}^{S}(L-r)\right] .
$$

It describes the amount of the unscreened spin at distance $R$ from the impurity site [18]. The impurity is completely screened by all bath electrons. To see this we consider $\mathcal{S}((L-$ 1)/2) on finite systems,

$$
\begin{aligned}
\mathcal{S}((L-1) / 2) & =\left\langle\Psi_{0}\left|\hat{S}_{d}^{z}\left(\hat{S}_{d}^{z}+\sum_{r=0}^{L-1} \hat{S}_{r, c}^{z}\right)\right| \Psi_{0}\right\rangle \\
& =\left\langle\Psi_{0}\left|\hat{S}_{d}^{z} \hat{S}^{z}\right| \Psi_{0}\right\rangle=0
\end{aligned}
$$

because $\left|\Psi_{0}\right\rangle$ is an eigenstate of the operator $\hat{S}^{z}$ for the total spin in the $z$ direction with eigenvalue zero.

\section{Spin correlations in two-chain geometry}

For the first site of the chain we have

$$
C_{d c}^{S}(0)=\frac{1}{4}\left\langle\Psi_{0}^{C}\left|\left(\hat{n}_{\uparrow}^{d}-\hat{n}_{\downarrow}^{d}\right)\left(\hat{C}_{0, \uparrow}^{+} \hat{C}_{0, \uparrow}-\hat{C}_{0, \downarrow}^{+} \hat{C}_{0, \downarrow}\right)\right| \Psi_{0}^{C}\right\rangle,
$$

where we used Eq. (39) and the normalization of $\left|\Psi_{0}^{S}\right\rangle$.

For the spin correlation function between the impurity site and a bath site at distance $1 \leqslant r \leqslant(L-1) / 2$ we use inversion symmetry (42) to write

$$
\begin{aligned}
C_{d c}^{S}(r) & =\left\langle\Psi_{0}\left|\hat{S}_{d}^{z} \hat{S}_{r}^{z}\right| \Psi_{0}\right\rangle \\
& =\frac{1}{2}\left\langle\Psi_{0}\left|\hat{S}_{d}^{z}\left(\hat{S}_{r}^{z}+\hat{S}_{L-r}^{z}\right)\right| \Psi_{0}\right\rangle \\
& =\frac{1}{8}\left\langle\Psi_{0}^{C}\left|\left(\hat{n}_{\uparrow}^{d}-\hat{n}_{\downarrow}^{d}\right)\left(\hat{C}_{r, \uparrow}^{+} \hat{C}_{r, \uparrow}-\hat{C}_{r, \downarrow}^{+} \hat{C}_{r, \downarrow}\right)\right| \Psi_{0}^{C}\right\rangle,
\end{aligned}
$$


where we used the mapping onto the chain operators in the second step,

$$
\hat{c}_{r, \sigma}^{+} \hat{c}_{r, \sigma}+\hat{c}_{L-r, \sigma}^{+} \hat{c}_{L-r, \sigma}=\hat{C}_{r, \sigma}^{+} \hat{C}_{r, \sigma}+\hat{S}_{r, \sigma}^{+} \hat{S}_{r, \sigma},
$$

and the factorization (39) in the last step; recall that the $S$ electron system is a paramagnetic Fermi sea, $\left\langle\Psi_{0}^{S}\right| \hat{S}_{r, \uparrow}^{+} \hat{S}_{r, \uparrow}-$ $\hat{S}_{r, \downarrow}^{+} \hat{S}_{r, \downarrow}\left|\Psi_{0}^{S}\right\rangle=0$.

Equations (45) and (46) must be evaluated using DMRG, in general. For $U=0$, the ground-state energy and the spin correlation function can be evaluated analytically to a large extent, as we show next.

\section{NONINTERACTING SIAM}

It is instructive to discuss the noninteracting SIAM. Moreover, it provides the basis for the Gutzwiller approach in Sec. IV. We defer the details of the derivation to the Supplemental Material [27] and merely summarize the relevant results.

\section{A. Ground-state energy}

The ground-state energy sums the band contribution and the energy of the doubly occupied bound state. The total energy reads [27]

$$
\begin{aligned}
e_{0}(V)= & e_{0}^{\text {band }}(V)+e_{0}^{\mathrm{b}}(V) \\
= & \frac{1}{2 \pi}\left[-\pi+2 v_{+} \arctan \left(\frac{1}{v_{-}}\right)\right. \\
& \left.+v_{-} \ln \left(\frac{v_{+}-1}{v_{+}+1}\right)\right]+\left(1-v_{+}\right),
\end{aligned}
$$

where

$$
v_{ \pm}(V) \equiv v_{ \pm}=\frac{\sqrt{\sqrt{1+64 V^{4}} \pm 1}}{\sqrt{2}} .
$$

The small- $V$ expansion becomes

$$
e_{0}^{\text {small }}(V)=\frac{4 V^{2}}{\pi}\left[\ln \left(V^{2}\right)+\ln (2)-1\right]-4 V^{4} .
$$

Corrections are of the order $V^{6} \ln \left(V^{2}\right)$. For $V=0.1$, the approximate formula works very well. We have $e_{0}(0.1)=$ -0.06291 whereas the approximation gives $e_{0}^{\text {small }}(0.1)=$ -0.06294 , with a relative error of less than one per mill.

To determine the Gutzwiller variational energy we also need the derivative of the ground-state energy. We have

$$
\begin{aligned}
e_{0}^{\prime}(x)= & \frac{4 x}{\pi\left[v_{+}(x)^{2}+v_{-}(x)^{2}\right]}\left[2 \pi v_{-}(x)\left(\frac{\operatorname{arccot}\left[v_{-}(x)\right]}{\pi}-1\right)\right. \\
& \left.+v_{+}(x) \ln \left(\frac{v_{+}(x)-1}{v_{+}(x)+1}\right)\right] .
\end{aligned}
$$

For small $x$ this reduces to

$$
e_{0}^{\prime}(x \ll 1) \approx(8 x / \pi) \ln \left(2 x^{2}\right) .
$$

\section{B. Magnetization and zero-field magnetic susceptibility}

We introduce the magnetic energy scale $B_{\text {imp }} \equiv B=$ $\left(g \mu_{\mathrm{B}} / 2\right) \mathcal{H}$, where $\mathcal{H}$ is the external magnetic field at the impurity, and express the impurity magnetization $M(V, \mathcal{H})=$ $g \mu_{\mathrm{B}} m(V, B)$ in terms of the impurity spin in the $z$ direction,

$$
m(V, B)=\left\langle\hat{S}_{d}^{z}\right\rangle=\left(\left\langle\hat{n}_{d, \uparrow}-\hat{n}_{d, \downarrow}\right\rangle\right) / 2 .
$$

The magnetic susceptibility follows from

$$
\chi(V, B)=\frac{\partial M(\mathcal{H})}{\partial \mathcal{H}}=\left(\frac{g \mu_{\mathrm{B}}}{2}\right)^{2} \frac{\partial[2 m(V, B)]}{\partial B} .
$$

We give closed expressions for $m(V, B)$ and $\chi(V, B)$ for the noninteracting SIAM in one dimension.

\section{Magnetization}

For the one-dimensional noninteracting SIAM we find for a magnetic field that acts solely at the impurity [27]

$$
\begin{aligned}
2 m(V, B)= & Z\left[v_{\mathrm{b}}(V, B)\right]-Z\left[v_{\mathrm{b}}(V,-B]\right. \\
& +\sum_{\sigma_{n}= \pm 1} \int_{-1 / 2}^{0} \frac{d \omega}{\pi} \frac{\sigma_{n} \Gamma \sqrt{1-4 \omega^{2}}}{\left(\omega+\sigma_{n} B\right)^{2}\left(1-4 \omega^{2}\right)+\Gamma^{2}}
\end{aligned}
$$

with $\Gamma=2 V^{2}$. Here, $v_{\mathrm{b}}(V, B)<-1 / 2$ is the energy of the bound state outside the band. It is the root of $P_{+}(\omega, B)$, i.e., $P_{+}\left(v_{\mathrm{b}}(V, B)\right)=0$, with

$$
P_{+}(\omega, B)=\omega+B+\frac{2 V^{2}}{\sqrt{4 \omega^{2}-1}} .
$$

Moreover, the weight of the bound state in the $d$-electron spectral function is given by

$$
Z\left[v_{\mathrm{b}}(V, B)\right]=\left[1-\frac{8 V^{2} v_{\mathrm{b}}(V, B)}{\left\{4\left[v_{\mathrm{b}}(V, B)\right]^{2}-1\right\}^{3 / 2}}\right]^{-1} .
$$

In general, the magnetization must be determined numerically from Eqs. (55) and (57).

\section{Small hybridizations}

In the limit $V \ll 1$, we ignore the bound-state contribution of order $V^{4}$, and simplify the magnetization to

$$
\begin{aligned}
m(V, B) & =\int_{-\infty}^{0} \frac{d \omega}{2 \pi}\left[\frac{\Gamma}{(\omega+B)^{2}+\Gamma^{2}}-\frac{\Gamma}{(\omega-B)^{2}+\Gamma^{2}}\right] \\
& =\int_{0}^{B} \frac{d \omega}{\pi} \frac{\Gamma}{\omega^{2}+\Gamma^{2}}=\frac{1}{\pi} \tan ^{-1}(B / \Gamma) .
\end{aligned}
$$

The width $\Gamma$ of the $d$-electron spectral function is the relevant energy scale for magnetic excitations.

For small hybridizations, the susceptibility becomes

$$
\chi(V, B)=\left(\frac{g \mu_{\mathrm{B}}}{2}\right)^{2} \frac{2}{\pi} \frac{\Gamma}{B^{2}+\Gamma^{2}}
$$

with the zero-field limit

$$
\chi_{0}(V)=\left(\frac{g \mu_{\mathrm{B}}}{2}\right)^{2} \frac{2}{\pi \Gamma} .
$$

As seen from Eq. (59), in the limit $V \rightarrow 0$ the magnetic susceptibility is proportional to the zero-field $d$-electron spectral function, $\mu(V, B) \propto D_{d, d, \sigma}(B)$ [27]. 


\section{External magnetic field for impurity and bath electrons}

For the case $B_{\text {imp }}=B_{\text {bath }} \equiv B$, the bound states are shifted in energy,

$$
v_{\mathrm{b}}(V, B)=-B-v_{+}(V) / 2,
$$

but their weights $Z\left[v_{\mathrm{b}}(V, B)\right]$ do not change because $v_{\mathrm{b}}(V, \pm B) \pm B=-v_{+}(V) / 2$ in both cases. The rigid shift in single-particle energies by the magnetic field also guarantees that the impurity remains half filled on average for all external fields. The impurity magnetization becomes $(B \ll W)$

$$
\begin{aligned}
2 \tilde{m}(V, B) & =\sum_{\tau= \pm 1} \int_{-\infty}^{0} \frac{V^{2} \rho_{0}(\omega+\tau B)}{(\omega+\tau B)^{2}+\left[\pi V^{2} \rho_{0}(\omega+\tau B)\right]^{2}} \\
& =2 \int_{0}^{B} \frac{d \omega}{\pi} \frac{\Gamma \sqrt{1-4 \omega^{2}}}{\omega^{2}\left(1-4 \omega^{2}\right)+\Gamma^{2}} .
\end{aligned}
$$

For small hybridizations, $\tilde{m}(V, B)$ reduces to the result for $m(V, B)$ in Eq. (58).

We show the impurity magnetization as a function of $B / \Gamma$ in Fig. 2. Only for $V=0.2$ and $B \gtrsim 2 \Gamma$, there is a discernible difference between the curves for $m(0.2, B)$, Eq. (55), where the external field is confined to the impurity, and $\tilde{m}(0.2, B)$, Eq. (62), where the external field polarizes all electrons. In both cases, the DMRG data, see Sec. V, faithfully reproduce the analytic results, within small errors resulting from finitesize effects.

The wide-band limit closely follows the result for the global magnetic field. This indicates that the difference between applying the external field locally or globally is mostly due to the polarization of the bound states for a local field. The bound states have a noticeable weight for $V=0.2$.

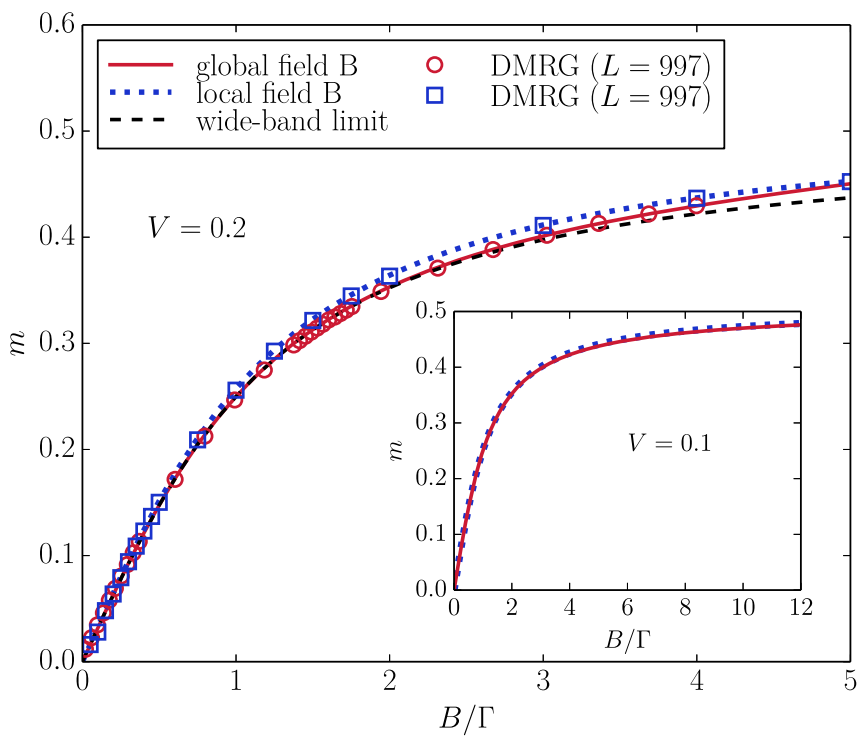

FIG. 2. Impurity magnetization for the noninteracting symmetric SIAM for $V=0.2$ as a function of $B=g \mu_{\mathrm{B}} \mathcal{H} / 2$. We show $m(0.2, B)$, Eq. (55) (local field, blue dotted line), $\tilde{m}(0.2, B)$, Eq. (62) (global field, red straight line), and the wide-band limit (58) (local field, black dashed line), together with the corresponding DMRG data (symbols, $L=997$ sites). Inset: Impurity magnetization for $V=0.1$.
Since the weight of the bound states is of the order $V^{4}$, their contribution is much smaller for $V=0.1$. Correspondingly, as seen from the inset of Fig. 2, the discrepancies between the magnetization curves for local and global external fields become very small. Since we shall work with $V \leqslant 0.1$ for the rest of the paper, we will restrict ourselves to purely local external magnetic fields, and shall safely ignore the influence of the magnetic field on the bath electrons.

\section{Spin correlation function \\ 1. General properties}

Starting from Eq. (41) we can use Wick's theorem and spin symmetry to show that

$$
C_{d c}^{S}(r)=-\frac{1}{2}\left|\left\langle\Phi_{0}\left|\hat{c}_{r, \uparrow}^{+} \hat{d}_{\uparrow}\right| \Phi_{0}\right\rangle\right|^{2} \equiv-\frac{1}{2} M_{r}^{2}
$$

for the ground state $\left|\Phi_{0}\right\rangle$ of the noninteracting SIAM. The matrix element is calculated in the Supplemental Material [27],

$$
\begin{aligned}
M_{r} & =\sqrt{\frac{1}{L}} \sum_{k} e^{-i k r}\left\langle\Phi_{0}\left|\hat{c}_{k, \uparrow}^{+} \hat{d}_{\uparrow}\right| \Phi_{0}\right\rangle \\
& =V \int_{0}^{\pi} \frac{d k}{\pi} \cos (k r) M[-\cos (k) / 2],
\end{aligned}
$$

where we took the thermodynamic limit and used $\epsilon(k)=$ $-\cos (k) / 2$ in one dimension. Since $M(\epsilon)$ is real, particle-hole symmetry leads to $M(-\epsilon)=M(\epsilon)$ so that the matrix element vanishes for odd sites, $M_{2 m-1}=0, m \geqslant 1$. For even sites we find the bound-state and band contributions [27]

$$
\begin{aligned}
M_{2 m}^{\mathrm{b}}= & -\frac{2 V Z(V)}{\sqrt{v_{+}^{2}-1}}\left(\sqrt{v_{+}^{2}-1}+v_{+}\right)^{-2 m}, \\
M_{2 m}^{\mathrm{band}}= & -\frac{2 V(-1)^{m}}{\pi} \int_{0}^{\pi} d y \frac{\cos (y / 2)}{\sin ^{2}(y)+64 V^{4}} \\
& \times\left[\sin (y) \cos (m y)+8 V^{2} \sin (m y)\right]
\end{aligned}
$$

with the pole frequency $\omega_{\mathrm{b}}=-v_{+} / 2$ and the pole weight

$$
Z(V)=\frac{1}{1+4 V^{2} v_{+} / v_{-}^{3}},
$$

and $v_{ \pm}$from Eq. (49).

\section{Small hybridizations}

The bound-state contribution $M_{r}^{\mathrm{b}}$ is of the order $V^{3}$ for small $V$, and becomes exponentially small for $r \gg 1 /\left(4 V^{2}\right)$. For small $V$ [17], the band contribution is dominated by the region $y \rightarrow 0$ in the integrand in Eq. (65). We thus approximate for small $V$

$$
\begin{aligned}
M_{2 m} & \approx-\frac{2 V}{\pi} \int_{0}^{\infty} d x \frac{x \cos \left(8 V^{2} m x\right)+\sin \left(8 V^{2} m x\right)}{x^{2}+1} \\
& =(-1)^{m} \frac{2 V}{\pi} e^{\alpha} \operatorname{Ei}(-\alpha), \quad \alpha=8 V^{2} m,
\end{aligned}
$$

where

$$
\operatorname{Ei}(x)=-\int_{-x}^{\infty} d t \frac{e^{-t}}{t}
$$




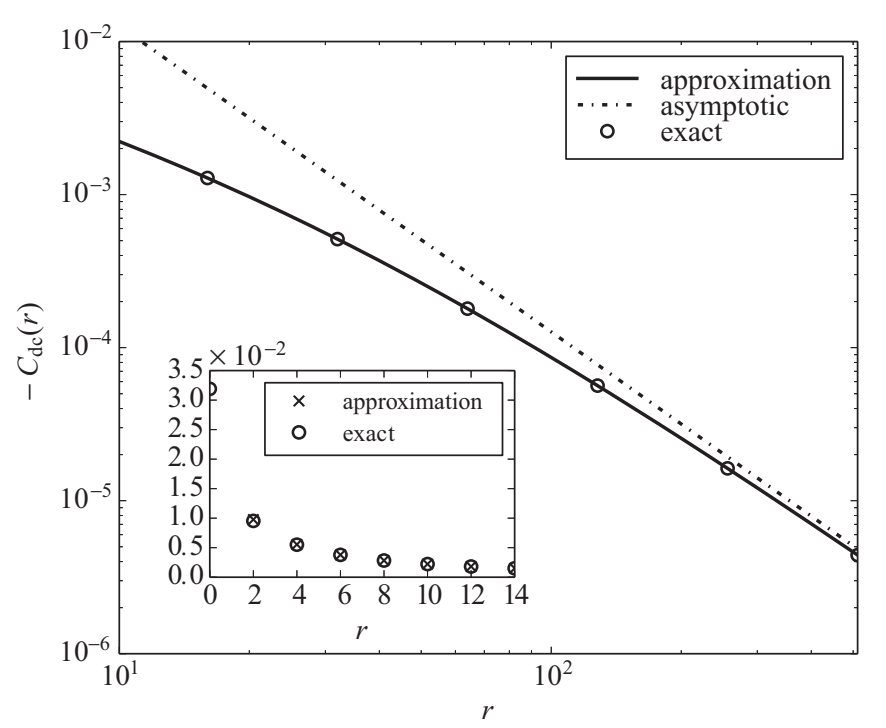

FIG. 3. Spin correlation function for the one-dimensional noninteracting symmetric SIAM for $V=0.1$ (circles) on a log-log scale. The analytic result (69) is shown as a straight line. The asymptotics (70) is shown as dash-dotted line, and the exact values (65) are shown as open symbols. Inset: Spin correlation function for small distances on a linear scale.

is the exponential integral. Thus, the spin correlation function approximately becomes $(m \neq 0)$

$$
C_{d c}^{S}(2 m) \approx-\frac{1}{2}\left[\frac{2 V}{\pi} e^{\alpha} \operatorname{Ei}(-\alpha)\right]^{2}, \quad \alpha=8 V^{2} m .
$$

In Figs. 3 and 4 we show the spin correlation function and the unscreened spin for the noninteracting symmetric SIAM

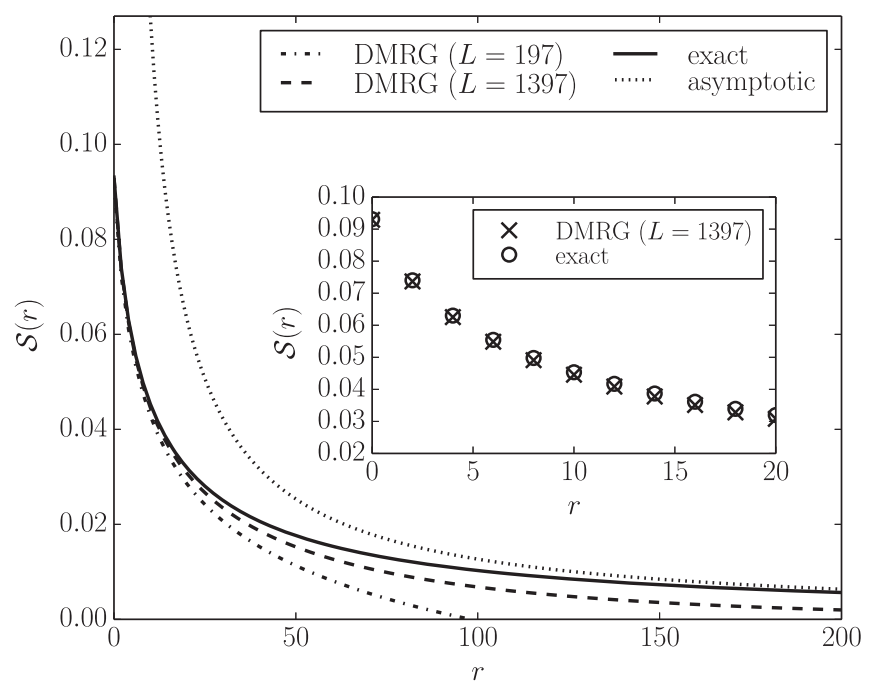

FIG. 4. Unscreened spin $\mathcal{S}(r)$ at distance $r$ from the impurity site, see Eq. (43), for the one-dimensional noninteracting symmetric single-impurity Anderson model for $V=0.1$. The analytic result based on Eq. (69) is shown as a solid line. The asymptotic result (72) is presented by a dotted line. The DMRG data for $L=197,1397$ sites are given by dashed lines; see Sec. V. Inset: Unscreened spin for small distances; DMRG data for $L=1397$ sites. in one dimension for $V=0.1$. As seen from Fig. 3, the spin correlation function decays to zero proportionally to $1 / \mathrm{m}^{2}$. The exact result (65) and the approximate formula (67) yield almost identical results, already for $m \geqslant 2$. For $m \geqslant 10$, the relative error is of the order $10^{-4}$ for $V=0.1$.

Correspondingly, the unscreened spin shown in Fig. 4 decays to zero proportionally to $1 / \mathrm{m}$. For small $V$, the screening is fairly inefficient and, correspondingly, the screening cloud extends very far into the host metal, even in the case of the noninteracting SIAM.

\section{Small hybridizations and large distances}

Here, we work out the long-range behavior of the spin correlation function. The asymptotic regime is reached for $\alpha \gg 1$, i.e., for $m \gg 1 /\left(8 V^{2}\right)$, where $\exp (\alpha) \operatorname{Ei}(\alpha) \approx-1 / \alpha$ in Eq. (69). For the correlation function we find in this region

$$
C_{d c}^{S}\left(2 m \gg 1 /\left(4 V^{2}\right)\right) \approx-\frac{1}{2}\left(\frac{2 V}{\pi \alpha}\right)^{2}=-\frac{1}{32 \pi^{2} V^{2}} \frac{1}{m^{2}} .
$$

For the noninteracting symmetric SIAM in one dimension, the spin correlations between the impurity and a bath electron at site $2 m$ asymptotically decay proportionally to $1 /(2 m)^{2}$; see Fig. 3.

The matrix element $M_{2 m}$ at $\alpha=1\left[2 m=1 /\left(4 V^{2}\right)\right]$ is already very small, of the order $V^{2}$ in the asymptotic region. Nevertheless, the contribution to the screening is finite even for $V \rightarrow 0$. The spins for $|m|>1 /\left(8 V^{2}\right)(\alpha>1)$ contribute approximately

$$
\begin{aligned}
\frac{\Delta S}{C_{d d}} & \approx 8\left(\frac{2 V}{\pi}\right)^{2} \int_{1 /\left(8 V^{2}\right)}^{\infty} d m\left[e^{\alpha} \operatorname{Ei}(-\alpha)\right]^{2} \\
& =\frac{4}{\pi^{2}} \int_{1}^{\infty} d \alpha\left[e^{\alpha} \operatorname{Ei}(-\alpha)\right]^{2} \approx 0.23
\end{aligned}
$$

The sites for $|m|>1 /\left(8 V^{2}\right)$ contribute about $25 \%$ to the total screening of the spin at the impurity site, where $C_{d d}=1 / 8$.

Indeed, for large distances $r=2 m$ from the impurity, the unscreened spin decays only proportionally to $1 / r$,

$$
\mathcal{S}(r \gg 1) \sim \frac{1}{16 \pi^{2} V^{2}} \frac{1}{r},
$$

as follows from Eq. (44) when we employ the EulerMaclaurin formula for the asymptotic expression (70). This is shown in Fig. 4.

\section{GUTZWILLER VARIATIONAL APPROACH}

In this section we define the Gutzwiller variational state and determine its variational parameters from minimizing the variational ground-state energy [23,24]. Moreover, we determine the variational magnetization, zero-field susceptibility, and spin-spin correlation function between the impurity site and the host electrons. 


\section{A. Ground-state energy}

\section{Definition}

The Gutzwiller wave function for the symmetric SIAM reads

$$
\begin{aligned}
\left|\Psi_{\mathrm{G}}\right\rangle= & {\left[\lambda_{d}\left(\hat{n}_{\uparrow}^{d} \hat{n}_{\downarrow}^{d}+\left(1-\hat{n}_{\uparrow}^{d}\right)\left(1-\hat{n}_{\downarrow}^{d}\right)\right)+\lambda_{\sigma}\left(\hat{n}_{\uparrow}^{d}\left(1-\hat{n}_{\downarrow}^{d}\right)\right.\right.} \\
& \left.\left.+\left(1-\hat{n}_{\uparrow}^{d}\right) \hat{n}_{\downarrow}^{d}\right)\right]\left|\Phi_{0}\right\rangle,
\end{aligned}
$$

where $\left|\Phi_{0}\right\rangle$ is a normalized single-particle product state.

The Gutzwiller wave function is normalized,

$$
\left\langle\Psi_{\mathrm{G}} \mid \Psi_{\mathrm{G}}\right\rangle=1,
$$

and symmetric,

$$
\left\langle\Psi_{\mathrm{G}}\left|\hat{n}_{d, \sigma}\right| \Psi_{\mathrm{G}}\right\rangle=\frac{1}{2},
$$

if we use a symmetric single-particle product state,

$$
\left\langle\Phi_{0}\left|\hat{n}_{d, \sigma}\right| \Phi_{0}\right\rangle=\frac{1}{2}
$$

and if we set

$$
\lambda_{d}^{2}=1-\sqrt{1-q^{2}}, \quad \lambda_{\sigma}^{2}=2-\lambda_{d}^{2}=1+\sqrt{1-q^{2}} .
$$

Here, we introduced the remaining variational parameter $0 \leqslant$ $q \leqslant 1$ that characterizes the Gutzwiller wave function.

\section{Optimizing the variational parameters}

The Gutzwiller variational ground-state energy with respect to the energy of the bare band is the minimum of

$$
E_{\mathrm{var}}(q)=e_{0}(q V)+\frac{U}{4}\left(1-\sqrt{1-q^{2}}\right)
$$

over the variational parameter $0 \leqslant q \leqslant 1$. Here, $e_{0}(V)$ is the ground-state energy of the noninteracting symmetric SIAM, $\hat{H}_{0}$ in Eq. (2); see Eq. (48). The minimum cannot be obtained analytically in general but we can derive an implicit equation.

The minimization condition $\left[d E_{\mathrm{var}}(q)\right] /(d q)=0$ leads to the equation

$$
U(q, V)=-2 \Gamma \sqrt{1-q^{2}} e_{0}^{\prime}(q V) /(q V)
$$

with $\Gamma=\pi \rho_{0}(0) V^{2}=\pi d_{0} V^{2}=2 V^{2}$ on a chain with nearestneighbor hopping. Therefore, we know $U(q, V)$ for every $0 \leqslant q \leqslant 1$. The variational ground-state energy is thus given implicitly by Eq. (78).

In Fig. 5 we show the Gutzwiller parameter as a function of $U / \Gamma$ for $V=0.1$, and compare to the analytic expression in the strong-coupling limit.

\section{Strong-coupling limit}

For strong couplings, we find $q \rightarrow 0$ so that we may use the small- $V$ expression to derive the variational ground-state energy analytically. Using Eq. (52) in Eq. (79) gives $q(U) \approx$ $q_{a}(U)$ with

$$
\left[q_{a}(U)\right]^{2}=\frac{1}{\Gamma} \exp \left(-\frac{\pi U}{16 \Gamma}\right),
$$

and the variational ground-state energy becomes

$$
E_{\mathrm{opt}}(q \ll 1, V) \approx-\frac{2}{\pi} \exp \left(-\frac{\pi U}{16 \Gamma}\right) \propto \exp \left(-\frac{1}{4 d_{0} J_{\mathrm{K}}}\right)
$$

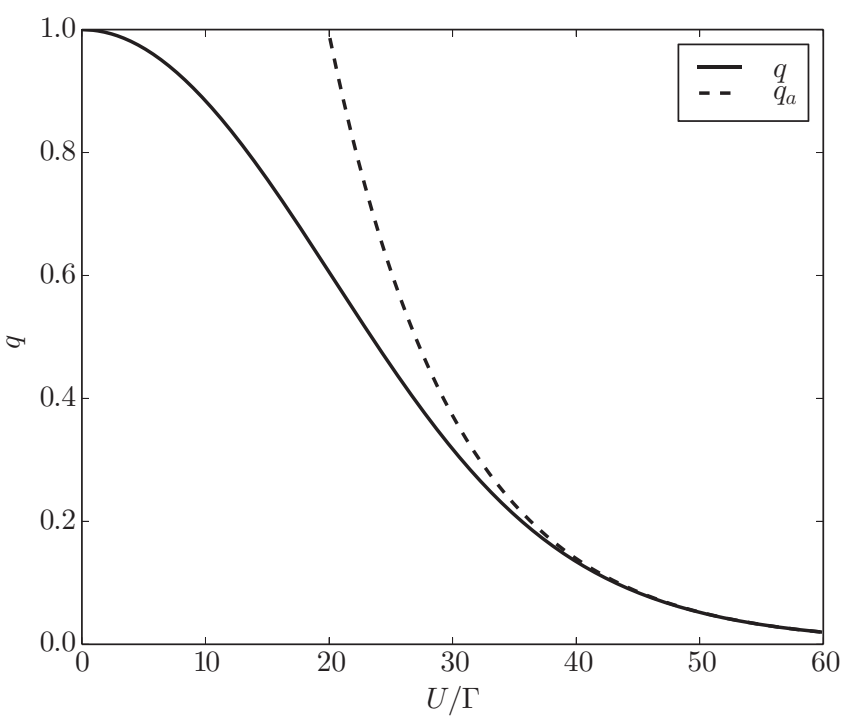

FIG. 5. Optimal Gutzwiller variational parameter as a function of $U / \Gamma$ for $V=0.1$ and the one-dimensional symmetric SIAM $\left(\Gamma=\pi d_{0} V^{2}=2 V^{2}\right)$. The asymptotic result (80) is shown with a dashed line.

with the Kondo energy $J_{\mathrm{K}}=4 V^{2} / U$.

The ground-state energy becomes exponentially small, corresponding to the exponentially small Abrikosov-Suhl resonance in the spectral function [3]. However, the Gutzwiller exponent is too small by a factor of two, $T_{\mathrm{K}} \propto \exp \left[-1 /\left(2 d_{0} J_{\mathrm{K}}\right)\right]$ [3]; i.e., the Gutzwiller approach overestimates the width of the resonance. As seen from Fig. 5, for $V=0.1$ the asymptotic behavior sets in around $U / \Gamma \approx 35$, for $q \lesssim 0.2$.

\section{B. Magnetization and magnetic susceptibility}

In the Gutzwiller variational approach, the impurity spin in the $z$ direction is given by

$$
m^{\mathrm{G}}(V, B)=\frac{\lambda_{\sigma}^{2}}{2}\left\langle\Phi_{0}\left|\hat{n}_{d, \uparrow}-\hat{n}_{d, \downarrow}\right| \Phi_{0}\right\rangle
$$

with $\lambda_{\sigma}$ from Eq. (77). Here, we keep a spin-independent $q$ factor and only consider the magnetic-field-induced changes in the single-particle product state $\left|\Phi_{0}\right\rangle$. Therefore, the Gutzwiller variational result for the magnetization can be obtained from the noninteracting expression by replacing $q$ with $q V$, see Sec. III B,

$$
m^{\mathrm{G}}(V, B)=\left(1+\sqrt{1-q^{2}}\right) m(q V, B) .
$$

The zero-field susceptibility in Gutzwiller theory reads

$$
\chi_{0}^{\mathrm{G}}(V, U)=\left(1+\sqrt{1-q^{2}}\right)\left(\frac{g \mu_{\mathrm{B}}}{2}\right)^{2} \frac{2}{\pi \Gamma} \frac{1}{q^{2}}
$$

so that the variational Wilson ratio becomes

$$
R^{\mathrm{G}}(V, U)=\frac{\chi_{0}^{\mathrm{G}}(V, U)}{\left(g \mu_{\mathrm{B}} / 2\right)^{2} D_{d, d}^{\mathrm{G}}(\omega=0)}=1+\sqrt{1-q^{2}} .
$$

Here, we used the fact that the Gutzwiller approach describes a Fermi liquid in which the density of states at the Fermi level is enhanced by a factor $1 / q^{2}$. Equation (85) shows that the Gutzwiller approach correctly reproduces the weak-coupling 
and strong-coupling limit, $R(U=0)=1$ and $R(U \gg \Gamma)=2$. In the strong-coupling limit, the Wilson ratio deviates from 2 algebraically in $1 / U$ due to the presence of charge fluctuations [3]. In contrast, the Gutzwiller Wilson ratio is exponentially close to 2 because the Gutzwiller approach does not describe charge excitations properly.

For strong couplings, the zero-field susceptibility becomes

$$
\Gamma \chi_{0}^{\mathrm{G}}(V, u \gg 1) \approx\left(\frac{g \mu_{\mathrm{B}}}{2}\right)^{2} \frac{4 \Gamma}{\pi} \exp \left(\frac{\pi u}{16}\right)
$$

with $u=U / \Gamma$. The Gutzwiller approach correctly reproduces the exponentially large zero-field susceptibility for strong interactions; see Sec. V D.

\section{Spin correlation function}

\section{Local correlation function}

The spin correlation function on the impurity site reads

$$
\begin{aligned}
C_{d d}^{S}(q) & =\frac{1}{4}-\frac{1}{2}\left\langle\Psi_{\mathrm{G}}\left|\hat{n}_{d, \uparrow}+\hat{n}_{d, \downarrow}\right| \Psi_{\mathrm{G}}\right\rangle \\
& =\frac{1}{4}-\frac{\lambda_{d}^{2}}{8}=\frac{1+\sqrt{1-q^{2}}}{8} .
\end{aligned}
$$

The value for the on-site spin correlation correctly interpolates between the itinerant limit, $C_{d d}^{S}(q=1)=1 / 8$, and the atomic limit, $C_{d d}^{S}(q=0)=1 / 4$.

\section{Correlation function between impurity and bath sites}

We continue with the spin correlation function between the impurity site and a bath site at distance $r$,

$$
\begin{aligned}
C_{d c}^{S}(q, r)= & \frac{1+\sqrt{1-q^{2}}}{4}\left\langle\Phi_{0}\right|\left(\hat{d}_{\uparrow}^{+} \hat{d}_{\uparrow}-\hat{d}_{\downarrow}^{+} \hat{d}_{\downarrow}\right) \\
& \times\left(\hat{c}_{r, \uparrow}^{+} \hat{c}_{r, \uparrow}-\hat{c}_{r, \downarrow}^{+} \hat{c}_{r, \downarrow}\right)\left|\Phi_{0}\right\rangle \\
= & -\frac{1+\sqrt{1-q^{2}}}{2}\left|\left\langle\Phi_{0}\left|\hat{c}_{r, \uparrow}^{+} \hat{d}_{\uparrow}\right| \Phi_{0}\right\rangle\right|^{2},
\end{aligned}
$$

where we applied spin symmetry and Wick's theorem in the last step. The matrix element is evaluated in Sec. III C, and we merely have to replace $V \rightarrow(q V)$ in all expressions there.

\section{INTERACTING SIAM}

In this section we compare our Gutzwiller variational results to those from the DMRG method that provides essentially exact numerical data for the SIAM on large rings. For comparison we also include results from magnetic HartreeFock theory, as derived in the Supplemental Material [27], and compare to the ground-state energy from the Bethe ansatz solution [32].

\section{A. DMRG method}

We study the symmetric SIAM in the effective single-chain representation (38) using the DMRG method. The mapping leads to an effective system size that is about half of the ring size, and it provides open boundary conditions that are more favorable for the DMRG method than periodic boundary conditions $[33,34]$. However, particle-hole symmetry is recovered only in the thermodynamic limit, $L \rightarrow \infty$.

\section{Technicalities}

We study the effective Hamiltonian of $C$ electrons on a chain up to a system of 700 sites, which corresponds to $L=$ 1397 in the ring geometry. This allows us to study systems with periodic boundary conditions that are three times longer than used in previous studies with open boundary conditions [18]. The accuracy of the calculations is controlled using the dynamic block-state selection (DBSS) scheme [35,36]. Setting the control parameter to $\chi=10^{-5}$, the truncation error yields around $10^{-7}$ while the number of maximally kept DMRG block states can grow up to $M=5000$ for large system sizes. For strong interactions, we target multiple states to stabilize convergence.

On finite lattices, the calculation of the magnetization as a function of a globally applied field $\mathcal{H}$ is more subtle because $S^{z}$ is a good quantum number. Therefore, the spin quantum number $S^{z}$ changes from $S^{z}=0$ for $\mathcal{H}=0$ to $S^{z}=1,2,3, \ldots$ for increasing external fields in steps of $g \mu_{\mathrm{B}} \mathcal{H}_{n}$ when

$$
g \mu_{\mathrm{B}} \mathcal{H}_{n}=E_{0}\left(S^{z}=n\right)-E_{0}\left(S^{z}=n-1\right)
$$

for $n=1,2,3, \ldots$ Thus, the impurity magnetization $\tilde{m}(V, B)$ is recorded only at discrete values of the external field whereby expectation values are calculated with the ground state for $S^{z}=n$. Since the energy differences are of the order $1 / L$, the smallest accessible magnetic energy scale is of the order $W / L$. In this work, we include only the results for $U=0, V=0.2$, see Fig. 2, to demonstrate the applicability of the approach.

For most of the results below, we apply the magnetic field only at the impurity. Since $\hat{P}$ in the Hamiltonian (2) is not conserved, standard DMRG ground-state calculations provide the results for the impurity magnetization.

\section{Tests}

To test the accuracy of our open-chain approach, in Fig. 6 we show the finite-size scaling of the impurity occupancy $n_{d, \sigma}(L)=\left\langle\hat{n}_{d, \sigma}\right\rangle$ for the open-chain SIAM (38) for various values of $U / \Gamma$ at $V=0.1$. It is seen that the occupation extrapolates to its value in the presence of particle-hole symmetry, $n_{d, \sigma}(\infty)=1 / 2$. For $U>0$, the electrons repel each other on the impurity. Thus, the Hubbard interaction suppresses charge fluctuations and shifts $n_{d, \sigma}(L)$ towards one-half already at small system sizes. Apparently, for numerical treatments the choice of open boundary conditions is favorable over the ring geometry because particle-hole symmetry holds also for finite system sizes. Of course, the boundary conditions play no role in the thermodynamic limit, as seen for the extrapolated impurity occupancy in Fig. 6.

As another test, we present the ground-state energy $\Delta E_{0}(U, V)$ as a function of inverse system size for $V=0.1$ and various values of the interaction strength $U / \Gamma$ in Fig. 7. Here, we measure the ground-state energy with respect to the case $V=0$,

$$
\Delta E_{0}(U, V)=E_{0}(U, V)-E_{0}(U, 0) ;
$$

i.e., we subtract the band contribution of the free host electrons and the term $-U / 4$ for the singly occupied impurity 


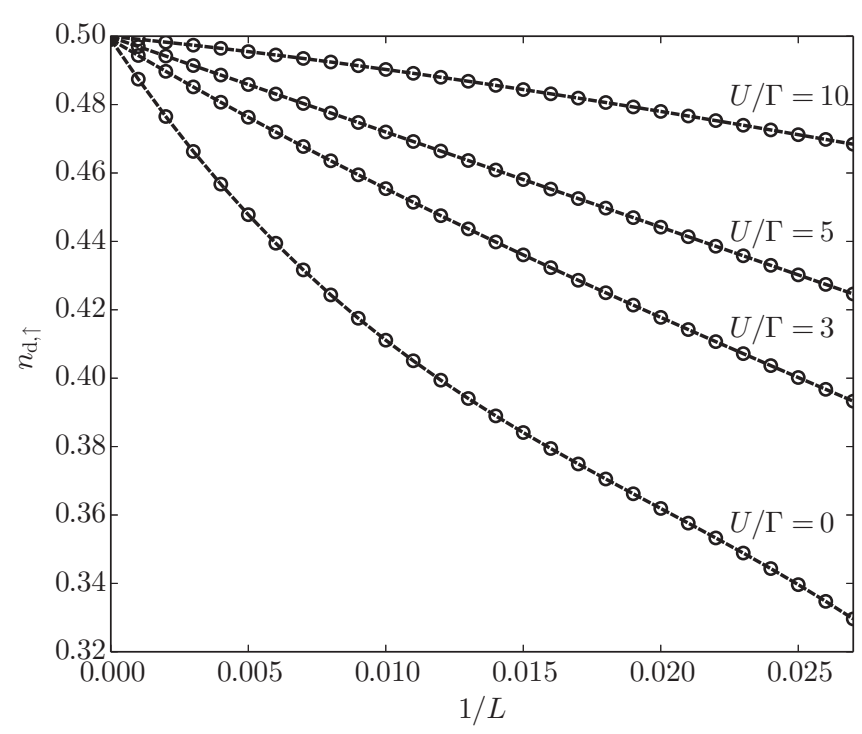

FIG. 6. DMRG results for the impurity occupation $n_{d, \uparrow}$ of the open-chain SIAM as a function of inverse system size $1 / L$ for various values of $U / \Gamma$ and $V=0.1\left(\Gamma=\pi d_{0} V^{2}=2 V^{2}\right)$. Lines are only guides to the eyes.

site. Therefore, $\Delta E_{0}(U, V)$ is of the order unity and tends to zero for large interaction strengths. Using a second-order polynomial fit in the inverse system size, the DMRG energies extrapolated to the thermodynamic limit coincide with the values from the Bethe ansatz. Note that the Bethe ansatz approach covers the wide-band limit, $U \ll W$, and also ignores corrections of order $V^{4}$. Therefore, the extrapolated DMRG energies are slightly below the Bethe ansatz energies.

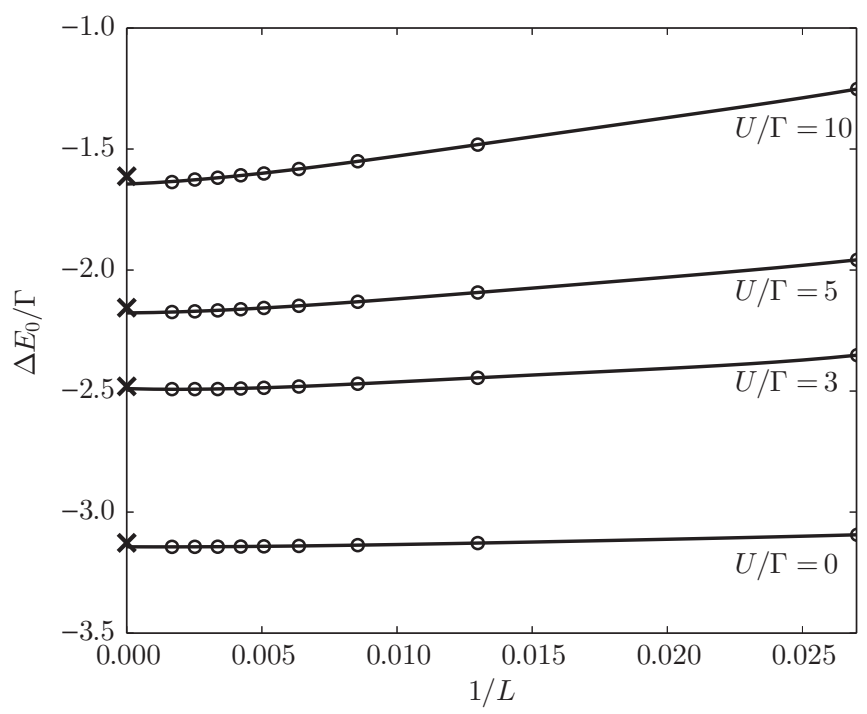

FIG. 7. DMRG result for the ground-state energy $\Delta E_{0}(U, V)$ of the one-dimensional symmetric SIAM as a function of inverse system size $1 / L$ for various values of $U / \Gamma$ and $V=0.1(\Gamma=$ $\pi d_{0} V^{2}=2 V^{2}$ ). The crosses denote the values from the Bethe ansatz, becoming exact in the wide-band limit. The solid lines represent the second-order polynomial fit.

\section{B. Ground-state energy}

\section{Small interaction strengths}

For the symmetric SIAM, the ground-state energy is known for weak coupling, $U \ll \pi \Gamma[3,37]$,

$$
\Delta E_{0}(U, V)=e_{0}(V)+\frac{U}{4}+\pi \Gamma \sum_{n=1}^{\infty}(-1)^{n} e^{(2 n)}(V)\left(\frac{U}{\pi \Gamma}\right)^{2 n},
$$

where $\Gamma=\pi d_{0} V^{2}=2 V^{2}$. Due to particle-hole symmetry, there are no odd-order corrections in the weak-coupling series beyond the Hartree term.

For $V \ll 1, e^{(2 n)}(V)$ weakly depends on $V$. We find (see Supplemental Material [27])

$$
\begin{aligned}
& e^{(2)}(0.1)=0.0374447, \\
& e^{(2)}(0.05)=0.0369271,
\end{aligned}
$$

in very good agreement with the analytical result obtained by Yamada [37],

$$
e^{(2)}(V=0)=\frac{1}{4}-\frac{7}{4 \pi^{2}} \zeta(3) \approx 0.0368608 .
$$

Moreover, the fourth-order coefficient is known to be very small, $e^{(4)}(V=0) \approx 0.0008$ [37].

The Gutzwiller approach leads to

$$
e_{\mathrm{G}}^{(2)}(V)=\frac{\pi \Gamma}{\left[-32 V e_{0}^{\prime}(V)\right]} \approx-\frac{\pi^{2}}{128 \ln \left(2 V^{2}\right)} .
$$

In contrast to the exact expression, the prefactor of the secondorder term vanishes logarithmically for $V \rightarrow 0$. For $V=0.1$, we find $e_{\mathrm{G}}^{(2)}(0.1)=0.01943$, about half of the exact value in Eq. (92). The paramagnetic Fermi sea remains the HartreeFock ground state until magnetic order sets in at about $U_{\mathrm{c}, \mathrm{HF}} \approx$ $\pi \Gamma$. Therefore, there is no second-order term in the groundstate energy in Hartree-Fock theory.

\section{Wide-band limit}

For small hybridizations and $V \ll U \ll W$, the SIAM can be solved analytically using the Bethe ansatz because the dispersion relation of the host electrons can be linearized around the Fermi wave vector [19-22]. For the symmetric SIAM in the absence of a magnetic field, the ground-state energy can be calculated analytically [32],

$$
\frac{\Delta E_{0}^{\mathrm{BA}}\left(U_{\mathrm{BA}}, V_{\mathrm{BA}}\right)}{t_{\mathrm{BA}}}=\frac{U_{\mathrm{BA}}}{2}+\int_{-\infty}^{A^{2}} d \Lambda 2 x(\Lambda) \sigma_{S}(\Lambda)
$$

with

$$
\begin{aligned}
x(\Lambda)= & -\frac{\sqrt{2}}{2} \sqrt{\Lambda+\sqrt{\Lambda^{2}+U_{\mathrm{BA}}^{2} V_{\mathrm{BA}}^{4} / 4}}, \\
\sigma_{S}(\Lambda)= & \int_{-\infty}^{\infty} \frac{d k}{4 \pi} \frac{V_{\mathrm{BA}}^{2}}{\left(k+U_{\mathrm{BA}} / 2\right)^{2}+V_{\mathrm{BA}}^{4} / 4} \\
& \times \frac{1}{U_{\mathrm{BA}} V_{\mathrm{BA}}^{2}} \operatorname{sech}\left[\frac{\pi\left(k^{2}-\Lambda\right)}{U_{\mathrm{BA}} V_{\mathrm{BA}}^{2}}\right],
\end{aligned}
$$

where $\operatorname{sech}(x)=1 / \cosh (x)$ is the hyperbolic secant function. The energy shift $U_{\mathrm{BA}} / 2$ takes our definition into account that 
$\Delta E_{0}(U, V)$ is measured with respect to the limit of vanishing hybridization, $\Delta E_{0}^{\mathrm{BA}}(U, 0)=0$.

Note that in Eq. (96) all energies are expressed in units of $t_{\mathrm{BA}}$ so that the Fermi velocity is $v_{\mathrm{F}}^{\mathrm{BA}}=t_{\mathrm{BA}}$. In our energy units we have $v_{\mathrm{F}}=W / 2$ so that we must set $t_{\mathrm{BA}}=W / 2 \equiv 1 / 2$; i.e., we must scale all energies by a factor of two. Moreover, in the Hamiltonian used in the Bethe ansatz, only the symmetric linear combination of right-movers and left-movers couples to the impurity whereas the hybridization in the lattice Hamiltonian (5) is expressed in terms of left-movers and right-movers. This implies $V_{\mathrm{BA}} / t_{\mathrm{BA}}=2 \sqrt{2} \mathrm{~V} / W$ and $U_{\mathrm{BA}}=2 U / W$ in our energy units.

We adjust the bandwidth cutoff parameter $A$ to reproduce the ground-state energy (50) of the noninteracting SIAM to orders $V^{2} \ln \left(V^{2}\right)$ and $V^{2}$. For $A=2 e$ we indeed find $\Delta E_{0}^{\mathrm{BA}}(0, V)=\left(4 V^{2} / \pi\right)\left[\ln \left(V^{2}\right)+\ln (2)-1\right]+$ $O\left(V^{4}\right)$; see Eq. (50).

Ignoring terms of order $V^{4}$ and higher that are beyond the wide-band limit, the ground-state energy reads

$$
\begin{aligned}
\frac{\Delta E_{0}^{\mathrm{BA}}(U, V)}{\Gamma}= & \frac{u}{2}+\sum_{\sigma} \int_{-e / \Gamma}^{0} \frac{d p}{\pi} \frac{1}{\left(p+\sigma_{n} u / 2\right)^{2}+1} \\
& \times \int_{-\infty}^{\infty} \frac{d y}{\pi} \operatorname{sech}(y) \tilde{x}\left(p^{2}-2 u y / \pi, u\right), \\
\tilde{x}(\lambda, u)= & \frac{\sqrt{2}}{2} \sqrt{\lambda+\sqrt{\lambda^{2}+u^{2}}}
\end{aligned}
$$

with $\Gamma=2 V^{2}$ and $u=U / \Gamma$. These expressions are amenable to a numerical evaluation of the integrals.

To extract the limiting behavior and to show the equivalence with the Hartree-Fock energy for $U / \Gamma \gg 1$, we write

$$
\Delta E_{0}^{\mathrm{BA}}(U, V)=\Delta E_{0}^{\mathrm{BA}, 1}(U, V)+\Delta E_{0}^{\mathrm{BA}, 2}(U, V),
$$

where

$$
\frac{\Delta E_{0}^{\mathrm{BA}, 1}(U, V)}{\Gamma}=\frac{u}{2}+\sum_{\sigma} \int_{-e / \Gamma}^{0} \frac{d p}{\pi} \frac{p}{\left(p+\sigma_{n} u / 2\right)^{2}+1}
$$

and

$$
\begin{aligned}
\frac{\Delta E_{0}^{\mathrm{BA}, 2}(U, V)}{\Gamma}= & \sum_{\sigma} \int_{-\infty}^{0} \frac{d k}{\pi} \frac{u}{\left(k \sqrt{u}+\sigma_{n} u / 2\right)^{2}+1} \\
& \times \int_{-\infty}^{\infty} \frac{d y}{\pi} \operatorname{sech}(y) X\left(k^{2}-2 y / \pi\right)
\end{aligned}
$$

with

$$
X(\lambda)=-k+\frac{\sqrt{2}}{2} \sqrt{\lambda+\sqrt{\lambda^{2}+1}} .
$$

The second term gives for $u \gg 1$

$$
\frac{\Delta E_{0}^{\mathrm{BA}, 2}(U \gg \Gamma, V)}{\Gamma}=\frac{\pi}{u}+O\left(1 / u^{2}\right) .
$$

The first term is equivalent to the Hartree-Fock expression in the limit $m \rightarrow 1 / 2$ that is reached for $U / \Gamma \gg 1$; see Supplemental Material [27]. Moreover, the integral is readily evaluated and gives in the intermediate-coupling regime

$$
\begin{aligned}
\left(\Gamma=2 V^{2} / W \ll U \ll W\right) & \ll E_{0}^{\mathrm{BA}, 1}(U, V) \\
\frac{\Delta}{\Gamma} & \frac{2}{\pi} \ln (\Gamma / e)+\frac{u}{2}\left[1-\frac{2}{\pi} \tan ^{-1}(u / 2)\right] \\
& +\frac{1}{\pi} \ln \left(1+u^{2} / 4\right)
\end{aligned}
$$

It is seen that the ground-state energy increases logarithmically, i.e., as a function of $\ln (u)$, in the intermediate-coupling regime [32].

One may wonder whether or not the Kondo energy scale can be extracted from the Bethe ansatz energy expression (97). Indeed, the region $\left[|p+u / 2| \leqslant v_{1},\left|y-p^{2} \pi /(2 u)\right| \leqslant\right.$ $v_{2}$ ] with $v_{1,2}$ of order unity gives rise to a contribution of the order of

$$
T_{\mathrm{L}}(U)=\Gamma \sqrt{\frac{u}{2}} \exp \left[-\frac{\pi u}{8}+\frac{\pi}{2 u}\right]
$$

with $u=U / \Gamma . T_{\mathrm{L}}(U)$ is proportional to the Kondo temperature for the symmetric SIAM in the strong-coupling limit [3]. Note, however, that the integration over all the $(p, y)$ region wipes out this term in the ground-state energy. It is only in magnetic properties that the energy scale $T_{\mathrm{L}}$ becomes visible [3]; see Sec. V C.

\section{Comparison}

In Fig. 8 we compare the Gutzwiller, Hartree-Fock, and Bethe ansatz energies for $V=0.01(\Gamma=0.0002)$. For such small hybridizations, DMRG calculations would require system sizes that are an order of magnitude larger because even at $U=0$ the relevant energy scale $\Gamma=2 V^{2} / W$ becomes very small. In Fig. 9 we show the ground-state energies for $V=0.1(\Gamma=0.02)$ from weak-coupling perturbation theory, Gutzwiller, Hartree-Fock, finite-size extrapolated data from

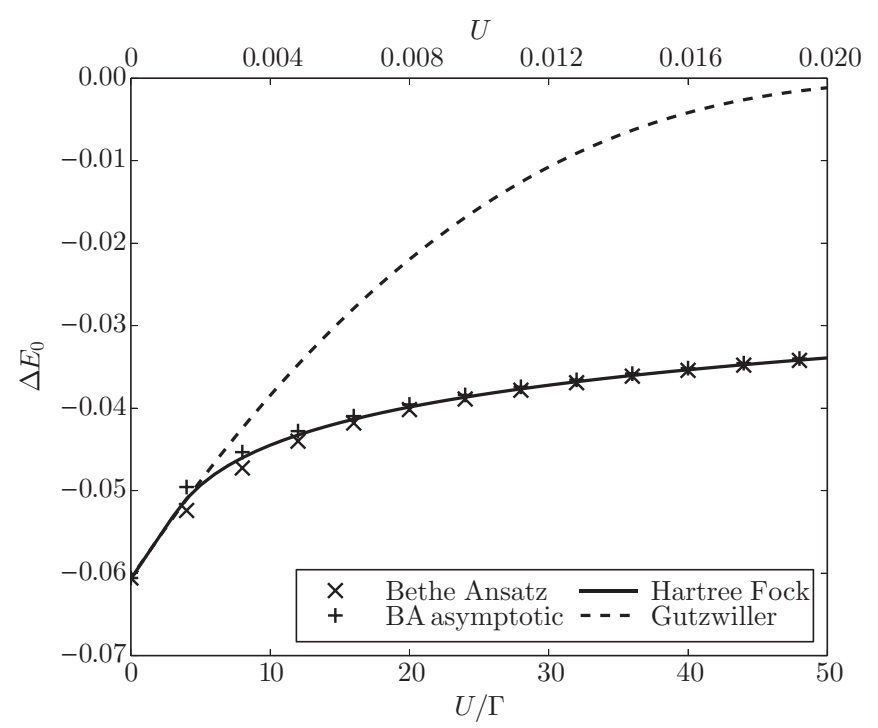

FIG. 8. Ground-state energy $\Delta E_{0}(U, V)$ for the symmetric SIAM in one dimension as a function $U / \Gamma$ for $V=0.01(\Gamma=$ $\pi d_{0} V^{2}=2 V^{2}=0.0002$ ). The full (dashed) lines display the Hartree-Fock (Gutzwiller) variational upper bound, the open symbols give the Bethe ansatz results from Eq. (97), and the crosses denote the asymptotic result (103). 


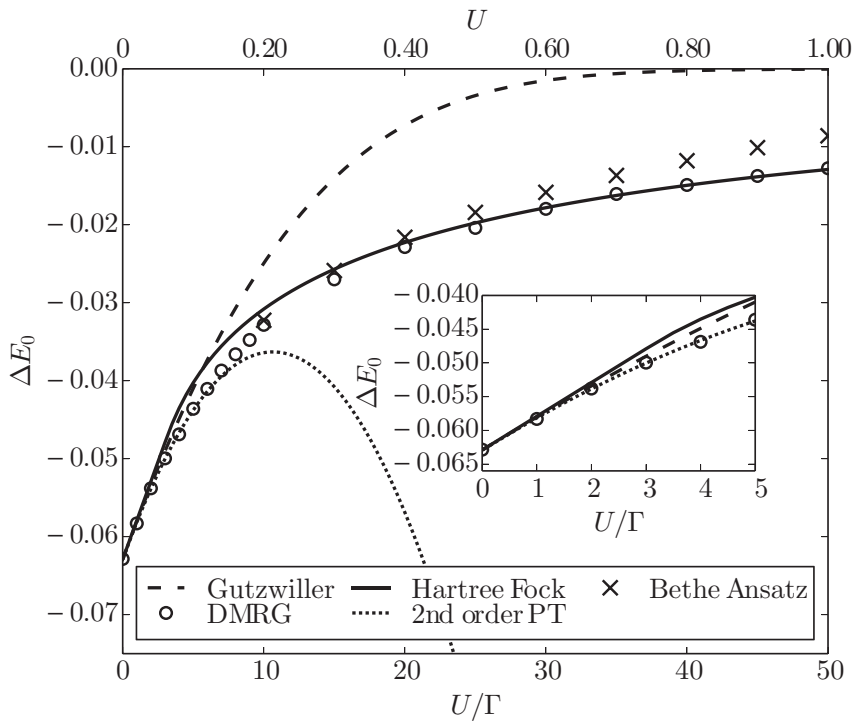

FIG. 9. Ground-state energy $\Delta E_{0}(U, V)$ for the symmetric SIAM on a ring as a function $U / \Gamma$ for $V=0.1\left(\Gamma=\pi d_{0} V^{2}=\right.$ $\left.2 V^{2}=0.02\right)$. The second-order weak-coupling result (92) is shown as a short-dashed line, the full (dashed) lines display the HartreeFock (Gutzwiller) variational upper bound, the open symbols are the DMRG data, extrapolated to the thermodynamic limit, and the crosses give the Bethe ansatz values. Inset: Ground-state energy for small interaction strengths.

DMRG, and the Bethe ansatz. Since the Bethe ansatz approach covers the wide-band limit, $U \ll W$, the extrapolated DMRG energies and the Hartree-Fock energies lie below the Bethe ansatz energies, as becomes discernible at $V=0.1$ for $U / \Gamma \gtrsim 10$ in Fig. 9.

As seen from the two figures, the Gutzwiller energy curve deviates noticeably from the exact results for $U / \Gamma>10$. Like second-order perturbation theory, it provides a good estimate only for $U \lesssim 5 \Gamma$. At large interactions, the Gutzwiller variational energy becomes exponentially small. Since the wave function does not properly describe charge fluctuations, i.e., the Hubbard bands, the Gutzwiller variational energy bound is poor.

In Hartree-Fock theory, a magnetic moment is formed only for $U_{\mathrm{c}}^{\mathrm{HF}} \approx \pi \Gamma$, and the ground-state energy contains a cusp at $U_{\mathrm{c}}$. More importantly, above $U \approx 5 \Gamma$ the Hartree-Fock theory provides an excellent bound on the exact ground-state energy. For small hybridizations, the exact Bethe ansatz and DMRG energies are in almost perfect agreement with the HartreeFock upper bounds. Since the quasiparticle peak provides an exponentially small energy contribution for $U \gg \Gamma$, the energy is solely determined by the lower Hubbard band, which gives rise to a $\ln (u)$ increase of the ground-state energy; see Eq. (103). The result for $\Delta E_{0}^{\mathrm{BA}, 1}(U, V) / \Gamma$ is also shown in Fig. 8.

Obviously, the lower Hubbard band for $u \gg 1$ is qualitatively well captured by Hartree-Fock theory. Therefore, Hartree-Fock theory provides an excellent starting point for analytical theories such as the local-moment approach that covers both the high-energy and low-energy parts of the single-particle spectrum [10-12].

\section{Magnetization and magnetic susceptibility}

The Bethe ansatz permits the exact calculation of the impurity magnetization in the presence of a magnetic field on the impurity. Hereby, it is implicitly understood that the effect of the magnetic field on the conduction electrons is negligibly small so that it does not make a difference whether or not the magnetic field is also applied to the bath electrons.

\section{Magnetization}

The analysis of the Bethe ansatz equations depends on the value of the external field $b=B / \Gamma$. First, region I, $b \leqslant b_{0}(u)$, covers the weak-field regime $b \rightarrow 0$ and the Kondo regime for $u \gg 1$. Region II, $b \geqslant b_{0}(u)$, covers the large-field regime $b \rightarrow \infty$. The magnetization and the magnetic susceptibility are continuous at $b=b_{0}(u)$. The boundary value is determined by $[\ln (e)=1]$

$$
\begin{aligned}
b_{0}(u) & =\sqrt{2 u} \frac{1}{\sqrt{\pi}} \sum_{n=0}^{\infty} \frac{1}{n !}\left(\frac{2 n+1}{2 e}\right)^{n+1 / 2} \frac{1}{(2 n+1)^{3 / 2}} \\
& \approx 0.398942 \sqrt{2 u} .
\end{aligned}
$$

For $u \rightarrow 0$, only region II exists, whereas in the Kondo limit, for $u \rightarrow \infty$, only region I remains.

Magnetization in region I. The magnetization and the magnetic field parametrically depend on each other. For $p \geqslant 0$, Tsvelik and Wiegmann give [21]

$$
b_{I}(p, u)=\sqrt{\frac{2 u}{\pi}} \sum_{n=0}^{\infty} \frac{1}{n !}\left(\frac{2 n+1}{2 e}\right)^{n+1 / 2} \frac{e^{-\pi(2 n+1) p}}{(2 n+1)^{3 / 2}}
$$

for the applied magnetic field so that $b_{I}(0, u)=b_{0}(u)$.

The impurity magnetization contains two terms,

$$
m_{I}(p, u)=m_{\mathrm{K}}(p, u)+m_{\mathrm{reg}}(p, u),
$$

namely, the "Kondo term" $m_{\mathrm{K}}(p, u)$ and the "regular term" $m_{\text {reg }}(p, u)$. We discuss them separately.

The Kondo term is given by $\left(\eta=0^{+}\right)[21,38]$,

$$
\begin{aligned}
m_{\mathrm{K}}(p, u)= & \frac{(-i)}{4 \pi^{3 / 2}} \int_{-\infty}^{\infty} \frac{d \omega}{\omega-i \eta}\left(\frac{i \omega+\eta}{e}\right)^{i \omega} \Gamma\left(\frac{1}{2}-i \omega\right) \\
& \times \exp \left\{-2 \pi i \omega\left[p-J^{-1}(u)\right]\right\},
\end{aligned}
$$

where

$$
J^{-1}(u)=\frac{u^{2}-4}{8 u}
$$

is the inverse Kondo coupling, and $\Gamma(x)$ denotes the Gamma function. The Kondo contribution cannot be obtained in weakcoupling perturbation theory because of the $1 / u$ singularity in the exponent. Moreover, it gives rise to a diverging zero-field susceptibility for $u \gg 1$; see Sec. V C 2 below.

The regular contribution reads

$$
\begin{aligned}
m_{\mathrm{reg}}(p, u)= & \frac{1}{\sqrt{\pi}} \sum_{n=0}^{\infty} \frac{1}{n !}\left(\frac{2 n+1}{2 e}\right)^{n+1 / 2} \frac{e^{-\pi p(2 n+1)}}{2 n+1} \\
& \times F\left(\frac{\pi(2 n+1)}{2 u}, u\right)
\end{aligned}
$$


with

$$
\begin{aligned}
F(a, u) & =\int_{-\infty}^{\infty} \frac{d y}{\pi} e^{-a y^{2}} \frac{1}{1+(i y+u / 2)^{2}} \\
& =\int_{0}^{\infty} \frac{2 d y}{\pi} e^{-a y^{2}} \frac{1-y^{2}+u^{2} / 4}{u^{2} y^{2}+\left(1-y^{2}+u^{2} / 4\right)^{2}} \\
& =e^{-a\left(1-u^{2} / 4\right)} \int_{0}^{a} \frac{d x}{\sqrt{\pi x}} \exp \left[x-\frac{a^{2} u^{2}}{4 x}\right]
\end{aligned}
$$

with the analytic expression

$$
\begin{aligned}
F(a, u)= & -e^{-a\left(1-u^{2} / 4\right)} \sin (a u) \\
& +e^{-a\left(1-u^{2} / 4\right)} \operatorname{Re}\left\{e^{i a u} \operatorname{Erfi}[\sqrt{a}(2-i u) / 2]\right\},
\end{aligned}
$$

where $\operatorname{Erfi}(x)$ is the complex error function. The analytic formula is helpful for the derivation of series expansions of $F(a, u)$ for small and large arguments $a$.

Magnetization in region II. For the applied magnetic field, Tsvelik and Wiegmann give [21]

$$
b_{I I}(p, u)=b_{0}(u)+\sqrt{\frac{u}{4 \pi}} \int_{0}^{\infty} \frac{d x}{x^{3 / 2}} \frac{\left(1-e^{-2 \pi p x}\right)}{\Gamma\left(\frac{1}{2}+x\right)}\left(\frac{x}{e}\right)^{x}
$$

for $p \geqslant 0$. The magnetization in region II is given by

$$
m_{I I}(p, u)=\frac{1}{2}-\frac{1}{2} \int_{0}^{\infty} \frac{d x}{\sqrt{\pi} x} \frac{e^{-2 \pi p x}}{\Gamma\left(\frac{1}{2}+x\right)}\left(\frac{x}{e}\right)^{x} F\left(\frac{\pi x}{u}, u\right) .
$$

As shown in the Supplemental Material [27], the result for the noninteracting SIAM is readily recovered from Eqs. (113) and (114). There, we also derive an explicit formula for the large-field limit,

$$
m_{I I}(b \gg \sqrt{u / \pi}) \approx \frac{1}{2}-\frac{1}{\pi b}+\frac{u}{2 \pi b^{2}}+\frac{4 \pi-12 u-3 \pi u^{2}}{12 \pi^{2} b^{3}}
$$

up to and including all terms of the order $1 / b^{3}$, and the lowenergy Kondo scale $T_{\mathrm{L}}(U)$ is absent for large fields.

In fact, there are no logarithmic terms to all orders of the $1 / b$ expansion because, for $b \gg 1$, both $b(p, u)$ and $m_{I I}(p, u)$ can be expressed in terms of a series with odd powers in the parameter $1 / \sqrt{z}$, where $z$ obeys $p=z-\ln (2 \pi e z) /(2 \pi)[21]$. Therefore, at large values of the external field, the impurity magnetization does not show any signs of the logarithmic Doniach-Šunjić-Hamann tails in the impurity spectral function [10-12,39].

We show the impurity magnetization as a function of the local external field for small and moderate interactions strengths in Fig. 10 and Fig. 11, respectively. The DMRG reproduces the magnetization curves very well, particularly at strong magnetic fields. DMRG requires very large system sizes to resolve the steep initial slope of the magnetization curves, especially for moderate to large interaction strengths. This behavior is reflected in the zero-field susceptibility that becomes exponentially large for large interactions in an exponentially narrow region of external fields, as we discuss next.

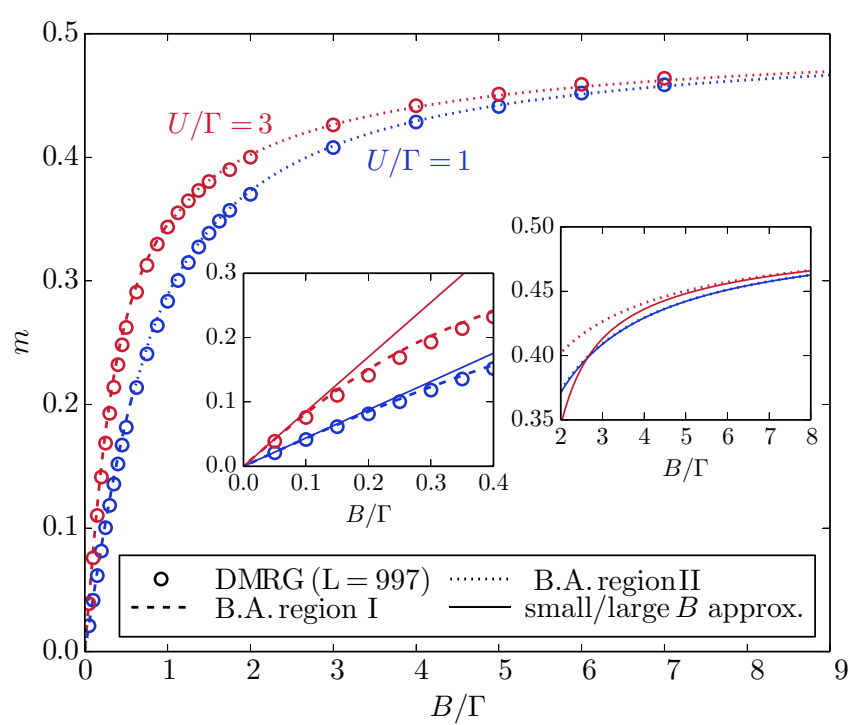

FIG. 10. Impurity magnetization $m(V=0.1, B)=\left\langle\hat{S}_{d}^{z}\right\rangle$ as a function of the external magnetic field $b=B / \Gamma=g \mu_{\mathrm{B}} \mathcal{H} /(2 \Gamma)$ for weak interactions, $u=U / \Gamma=1,3$, from the Bethe ansatz for the symmetric SIAM. The insets show the behavior for small and large fields with the small-field asymptotics (118) and the large-field asymptotics (115). The circles are DMRG results for rings with $L=997$ sites.

\section{Zero-field magnetic susceptibility}

For $b_{I}(p, u) \equiv b \rightarrow 0$, we have $p \rightarrow \infty$ in Eq. (106) so that we only retain the first term in the series. Thus,

$$
p=-\frac{1}{\pi} \ln \left(b \sqrt{\frac{\pi e}{u}}\right) \gg 1
$$

For the case $\left[p-J^{-1}(u)\right] \geqslant 0$, we may represent $m_{\mathrm{K}}(p, u)$ in terms of a sum by performing a contour integral in the lower

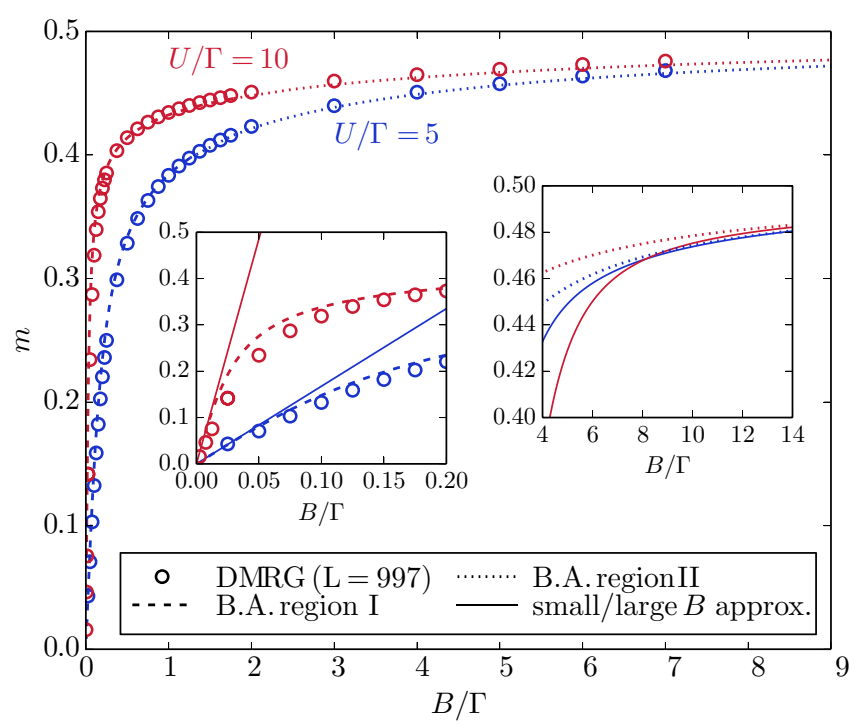

FIG. 11. Same as Fig. 10 for moderate couplings $u=5,10$. 
complex $\omega$ plane,

$$
\begin{aligned}
m_{\mathrm{K}}(p, u)= & \frac{1}{\sqrt{\pi}} \sum_{n=0}^{\infty} \frac{(-1)^{n}}{2 n+1} \frac{1}{n !}\left(\frac{2 n+1}{2 e}\right)^{n+1 / 2} \\
& \times \exp \left\{-\pi\left[p-J^{-1}(u)\right](2 n+1)\right\} .
\end{aligned}
$$

In this sum for the Kondo contribution and in the sum for the regular contribution, Eq. (110), we keep only the first term in the series and find $\left[m_{I}(p, u) \equiv m(b, u)\right]$

$$
m(b \rightarrow 0, u) \approx \frac{b}{\sqrt{2 u}} e^{\pi / J(u)}\left[1+e^{-\pi / J(u)} F(\pi /(2 u), u)\right]
$$

for small $b$, with corrections of the order $b^{3}$ [21].

Thus, the Bethe ansatz provides an explicit expression for the impurity susceptibility in the wide-band limit $[3,21,40]$,

$$
\begin{aligned}
\chi_{0}^{\mathrm{BA}}(U, V)= & \left(\frac{g \mu_{\mathrm{B}}}{2}\right)^{2} \frac{1}{T_{\mathrm{L}}(U)} \\
& \times\left[1+\int_{0}^{\pi /(2 u)} \frac{d x}{\sqrt{\pi x}} \exp \left(x-\frac{\pi^{2}}{16 x}\right)\right]
\end{aligned}
$$

with $u=U / \Gamma$ and $T_{\mathrm{L}}(U)$ from Eq. (104). Since the integral vanishes for $u \rightarrow \infty$, the exponential term gives the result in the Kondo limit.

We show the zero-field susceptibility in Fig. 12. As seen from Eqs. (104) and (119), the zero-field susceptibility increases exponentially as a function of $u$. This behavior is difficult to reproduce in DMRG because, as the magnetization is bounded from above, the magnetic-field region in which the susceptibility is exponentially large is exponentially small. Therefore, it is hard to calculate the zero-field susceptibility for $u \gtrsim 10$ from DMRG, and other numerical

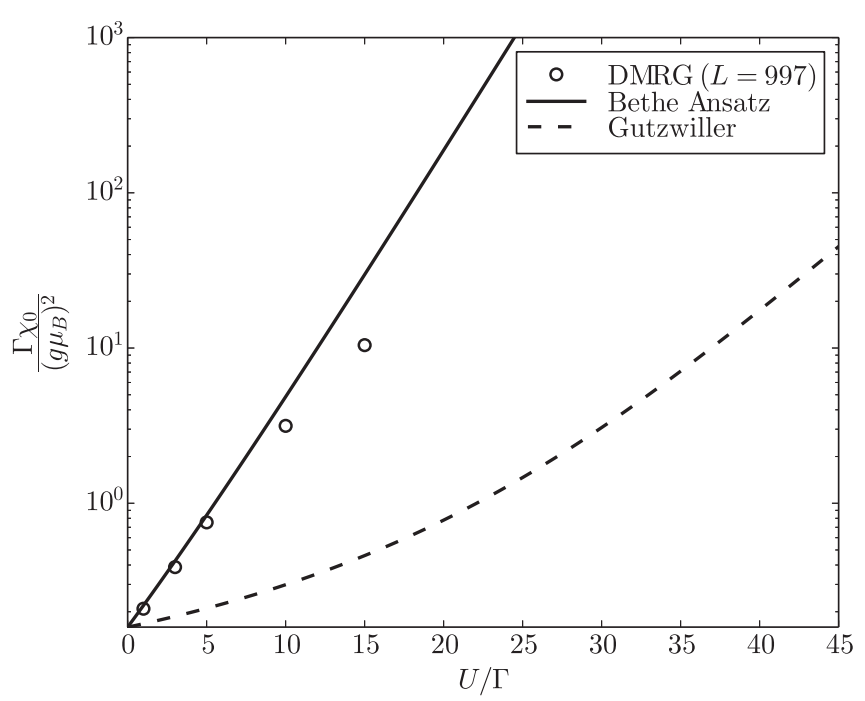

FIG. 12. Zero-field magnetic susceptibility, $\Gamma \chi_{0} /\left(g \mu_{\mathrm{B}}\right)^{2}$, as a function of the interaction strength $u=U / \Gamma$ from Bethe ansatz, Eq. (119), and the Gutzwiller variational approach, Eq. (84), for the SIAM, in comparison with DMRG results for $L=997$ sites. Note the logarithmic scale for the ordinate. methods such as the NRG must be employed for large interaction strengths. For $u=10,15$ we choose $B=0.0025 \Gamma$ and calculate $\chi_{0}^{\mathrm{DMRG}}(U, V=0.1)=\left(g \mu_{\mathrm{B}} / 2\right)^{2}[2 m(B, u) / B]$. As seen from Fig. 12, the agreement between the Bethe ansatz results and DMRG is very good for $u \lesssim 10$ and quite acceptable for $u \lesssim 15$, where the zero-field susceptibility is enhanced by more than a factor of 100 over its noninteracting value.

The Gutzwiller variational theory reproduces the exponential behavior of the zero-field susceptibility but with an exponent that is too small by a factor of two. Therefore, the Gutzwiller approach also underestimates the value of the zero-field spin susceptibility; see Fig. 12.

\section{Spin correlation function}

\section{Local moment}

In Fig. 13 we show the local moment on the impurity site, $C_{d d}=\left\langle\left(n_{d, \uparrow}-n_{d, \downarrow}\right)^{2}\right\rangle / 4$, from Gutzwiller, Hartree-Fock, and DMRG. The Gutzwiller approach provides a reasonable estimate for the local moment for all interaction strengths. However, it underestimates its value for weak interactions and slightly overestimates it in the strong-coupling limit.

Hartree-Fock theory uses the noninteracting Fermi-sea ground state for weak interactions, and starts with the interaction-driven buildup of the local moment at $U_{\mathrm{c}}^{\mathrm{HF}} \approx \pi \Gamma$. Similarly to the Hartree-Fock energy curve shown in Fig. 8, a kink in $C_{d d}^{\mathrm{HF}}(U)$ is observed at the critical Hartree-Fock interaction. For moderate to strong interactions, $U \gtrsim 5 \Gamma$, it provides an excellent estimate for the local spin correlation. For $U / \Gamma=5$ and for $U / \Gamma=50$, the magnitude of the local moment is of the same magnitude both in the Gutzwiller approach and in Hartree-Fock theory.

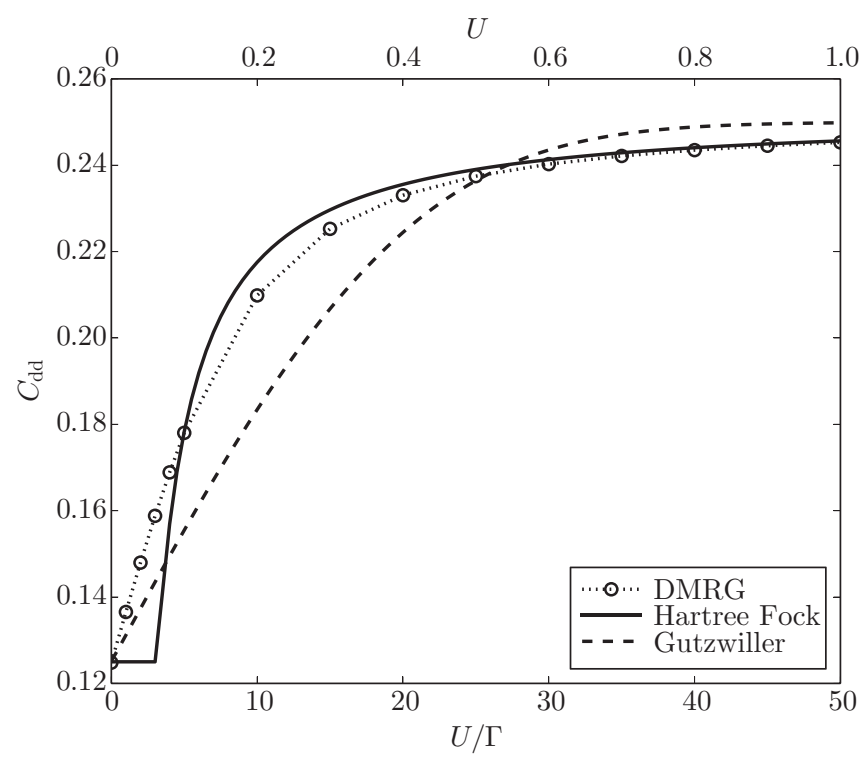

FIG. 13. Local spin correlation $C_{d d}=\left\langle\left(n_{d, \uparrow}-n_{d, \downarrow}\right)^{2}\right\rangle / 4$ for $V=0.1\left(\Gamma=2 V^{2}=0.02\right)$ as a function of $U / \Gamma=5$ in Gutzwiller and Hartree-Fock theory for the SIAM compared with DMRG results with $L=1397$ sites. 

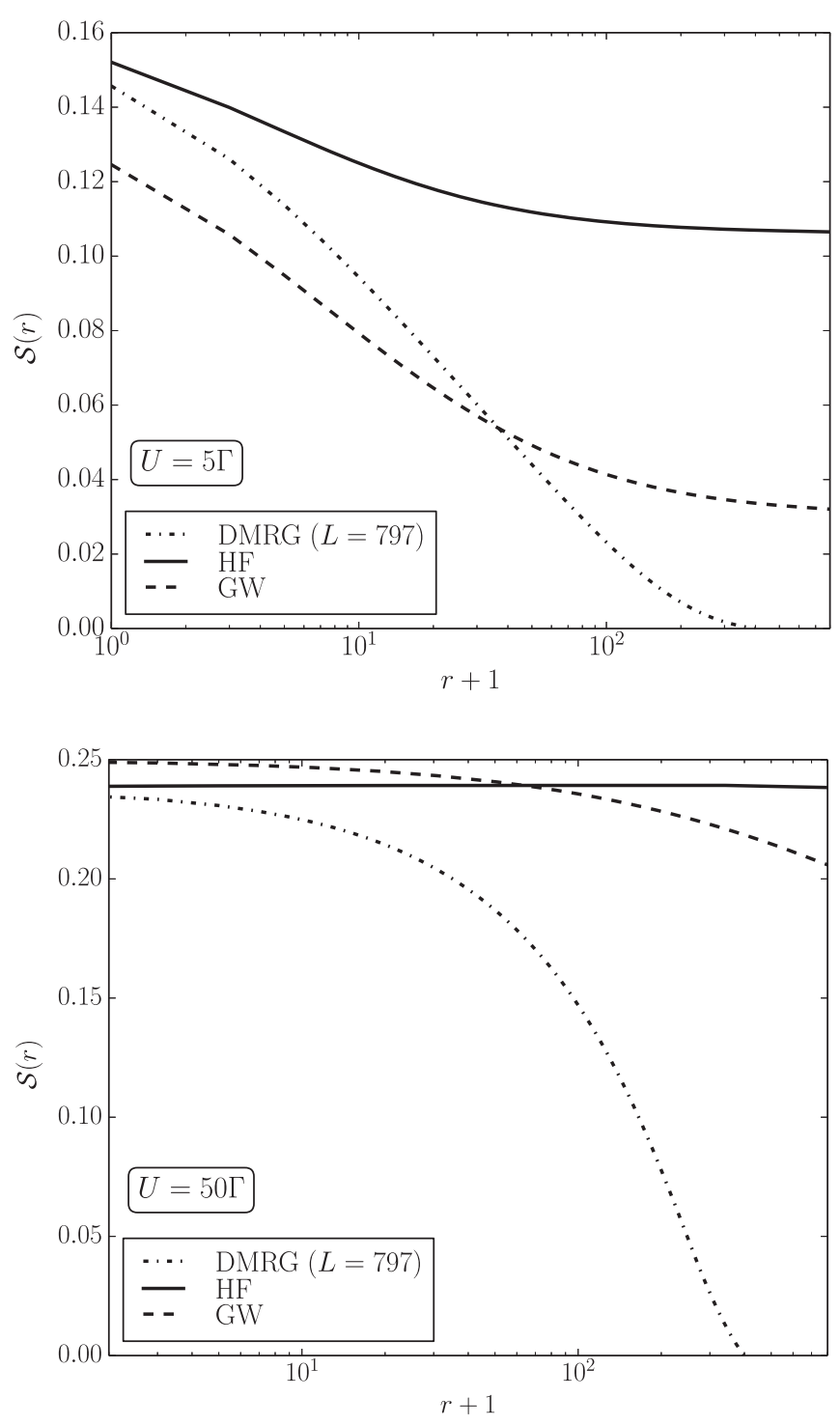

FIG. 14. Unscreened spin $\mathcal{S}(r)$ at distance $r$ from the impurity site, see Eq. (43), for $V=0.1\left(\Gamma=2 V^{2}=0.02\right), U / \Gamma=5$ (upper panel), and $U / \Gamma=50$ (lower panel). The Gutzwiller and magnetic Hartree-Fock results for the SIAM are compared with DMRG results with $L=797$ sites.

\section{Unscreened spin}

In Fig. 14 we show the unscreened $\operatorname{spin} \mathcal{S}(r)$ for $U / \Gamma=5$ (upper panel) and $U / \Gamma=50$ (lower panel) at $V=0.1(\Gamma=$ 0.02). Even for $U / \Gamma=5$, the asymptotic region is not yet reached for $L=797$ in $\mathrm{DMRG}$, where by construction the spin is screened at $r=(L-1) / 2=398$. For $U / \Gamma=5$, finitesize effects are unimportant up to $r \approx 30$. When the interaction is very large, $U=50 \Gamma$, finite-size effects dominate the DMRG data for all $r>1$.

Despite its failure to describe the ground-state energy properly, the Gutzwiller approach reproduces the mesoscopically large Kondo screening cloud. For $r \rightarrow \infty$, the impurity spin is perfectly screened, $\mathcal{S}^{\mathrm{G}}(r \rightarrow \infty)=0$, but the Kondo cloud extends over many thousands of sites even for moderately strong interactions, $U / \Gamma=5$. In contrast, in magnetic Hartree-Fock theory the screening is never complete, $\mathcal{S}^{\mathrm{HF}}(r \rightarrow \infty)>0$.
Therefore, among the three approaches discussed here, the Gutzwiller wave function provides the best qualitative description of the Kondo screening cloud for strong couplings.

\section{Fermi liquid regime}

The calculation of the static spin correlation function $C_{d c}(r)$ in Eq. (41) poses a difficult many-body problem. We separate the spin correlation function into its Fermi liquid contribution (dressed bubble) and a part that contains vertex parts in a diagrammatic approach,

$$
\begin{aligned}
C_{d c}^{S}(r) & =C_{d c}^{S, \mathrm{FL}}+C_{d c}^{S, x}, \\
C_{d c}^{S, \mathrm{FL}} & =-\frac{1}{2}\left|\left\langle\Psi_{0}\left|\hat{c}_{r, \uparrow}^{+} \hat{d}_{\uparrow}\right| \Psi_{0}\right\rangle\right|^{2}=-\frac{1}{2} M_{r}^{2} .
\end{aligned}
$$

The vertex part vanishes for the noninteracting case; see Eq. (63). For the Fermi liquid part, we used that the $d$-electron density is one-half and that the system is unpolarized.

Due to particle-hole symmetry for the translationally invariant system, the Fermi liquid contribution vanishes for odd sites, $C_{d c}^{S \text {, FL }}(2 m+1)=0$. The spin correlation function at odd sites remain small even for substantial interactions. However, from the DMRG data we infer that, for large interactions and intermediate length scales, the vertex term for even sites is (much) larger than the Fermi liquid contribution. In this regime, the ground state is very far from a single Slater determinant.

In the limit of very large distances and thus small excitation energies, we expect that the Fermi liquid picture description is applicable. For the Fermi liquid contribution we can write quite generally [27]

$$
\begin{aligned}
M_{2 m}= & -\frac{V}{\pi} \int_{-\pi / 2}^{0} d p \operatorname{Im}\{[\sin (2 m p)-i \cos (2 m p)] \\
& \left.\times \tilde{G}_{d, d}^{\mathrm{ret}}(\sin (p) / 2)\right\},
\end{aligned}
$$

where we neglected the contributions from the bound states because their contribution vanishes exponentially for large distances. Here, $\tilde{G}_{d, d}^{\mathrm{ret}}(\omega)$ is the exact retarded impurity Green's function.

At very large distances, only the region of small $p$ contributes to the integration because of the vastly oscillating sine and cosine functions. Thus, we may approximate the impurity spectral function by its Fermi liquid form,

$$
D_{d, d}(\omega) \approx \frac{1}{\pi} \frac{\Gamma_{*}}{\omega^{2}+\Gamma_{*}^{2}}
$$

with $\Gamma_{*} \propto T_{\mathrm{L}}$ with the Kondo scale from Eq. (104). Thus, we recover the result (70) for the decay of the spin correlation function at large distances,

$$
C_{d c}^{S}\left(2 m \gg 1 /\left(2 \Gamma_{*}^{2}\right)\right) \approx-\frac{V^{2}}{8 \pi^{2} \Gamma_{*}^{2}} \frac{1}{m^{2}} .
$$

The long-range decay of the correlation function is algebraic but, since $\Gamma_{*}$ is exponentially small, this decay only sets in at exponentially large length scales. The Gutzwiller approach reproduces this result qualitatively.

Note that the subtleties of the Kondo screening, e.g., the Doniach-Šunjić-Hamann tails in the impurity spectral function [10-12,39], contribute to the Kondo screening cloud $\mathcal{S}(r)$ for intermediate to large distances that are well below 
$1 / \Gamma_{*}^{2}$. The visualization of the screening cloud requires the calculation of a two-particle correlation function, which is very demanding; for a variational approach to the Kondo model, see Ref. [16].

\section{CONCLUSIONS}

In this work, we studied the ground-state energy, the impurity magnetization and susceptibility, and the Kondo screening cloud for the symmetric single-impurity Anderson model (SIAM) using the results from the Gutzwiller, magnetic Hartree-Fock, and DMRG variational approaches. We restricted our study to the case of a regular metal with a constant density of states around the Fermi energy; Kondo screening for other host density of states, e.g., in graphene, is studied in Refs. [25,41]. For the ground-state energy and magnetic properties, we compared our results to those from the Bethe ansatz that become exact in the wide-band limit. For further reference, we defer many technical details to the Supplemental Material [27].

Each of the three variational methods has its merits and limitations.

(1) The Hartree-Fock approach provides an excellent description of the ground-state energy for intermediate to strong couplings. However, since it displays a gap for (magnetic) excitations, Hartree-Fock theory fails to reproduce the large magnetic susceptibility for strong couplings. Concomitantly, it is unable to screen the impurity spin.

The Hartree-Fock theory correctly describes the charge excitations of the symmetric SIAM. This makes it the perfect starting point for more elaborate analytical approximations such as the local-moment approach that introduces the missing lowenergy spin-flip processes into the Hartree-Fock description [10-12].

(2) Gutzwiller theory provides a rather poor upper bound for the ground-state energy. However, it qualitatively describes the exponentially large magnetic zero-field susceptibility for strong couplings because it retains an exponentially small resonance in the impurity density of states in the Kondo limit. Consequently, the impurity spin is completely screened by the bath electrons at infinite distance from the impurity.

As an inherent Fermi liquid description, Gutzwiller theory correctly reproduces the long-range behavior of the Kondo cloud. However, for short and intermediate distances, its description of the Kondo cloud is too simple minded.

(3) The DMRG method is numerically highly accurate for finite systems. In this work, we map the SIAM on a ring onto a two-chain geometry with open boundary conditions, where we disregard the interchain coupling. Therefore, we can treat rings with up to $L=1400$ sites, and extrapolations of the ground-state energy to the thermodynamic limit are unproblematic. We see that the Bethe ansatz description is applicable for interactions up to about half the bandwidth even at $V=0.1 W$.

The intrinsic energy resolution is limited to $\Delta \omega=W / L$. Therefore, the DMRG encounters problems to resolve the Abrikosov-Suhl resonance in the impurity density of states in the strong-coupling limit, and a reliable description of the impurity magnetization and of the magnetic susceptibility is limited to moderate interaction strengths. Correspondingly,
DMRG properly describes the short-range region of the Kondo cloud but does not cover the long-distance asymptotics because the Kondo cloud in DMRG cannot exceed half the system size. The NRG is best suited to resolve small energy scales, and thus overcomes the DMRG limitations.

At the end of our presentation, we emphasize that the Kondo screening cloud is amazingly large, even in the noninteracting limit, for reasonably small hybridization strengths, e.g., $V=0.1$, and in one dimension, where a larger fraction of the bath electrons can couple to the impurity than in higher dimensions. This implies that magnetic impurities in metals can be correlated over mesoscopic distances.

Note, however, that this behavior depends on a number of assumptions, namely, (i) a perfect metallic host without impurities, (ii) zero temperature, and (iii) the Kondo regime, which is guaranteed for the SIAM by particle-hole and spin-flip symmetry for $U / \Gamma \gg 1$. Deviations from these exceptional conditions, especially a finite temperature, will drastically limit the range over which the impurity spin is screened.

Nevertheless, we can expect that two magnetic impurities in a metal can sense each others' presence over quite some distance so that they will bind into magnetic singlet (or triplet) pairs. An investigation of this pairing requires the analysis of the two-impurity Anderson model (TIAM); see, e.g., Ref. [42] for a recent Gutzwiller variational study, and references therein. A DMRG study of the TIAM is currently under way.

\section{ACKNOWLEDGMENTS}

This research has been supported in part by the Hungarian National Research, Development, and Innovation Office (NKFIH) through Grants No. K120569 and No. PD-17-125261 and the Hungarian Quantum Technology National Excellence Program (Project No. 2017-1.2.1-NKP-2017-00001). Ö.L. also acknowledges support from the Alexander von Humboldt Foundation.

\section{APPENDIX: CHAIN MAPPING USING THE LANCZOS CONSTRUCTION}

The Lanczos algorithm provides another way to derive the chain geometry from the ring geometry in Sec. II C. Dropping spin indices, we start from the seed state

$$
\left|\Psi_{0}\right\rangle=\hat{d}^{+}|\mathrm{vac}\rangle
$$

and find the Lanczos basis from

$$
\begin{aligned}
& \left|\Psi_{1}\right\rangle=\hat{H}_{0}\left|\Psi_{0}\right\rangle-a_{0}\left|\Psi_{0}\right\rangle=V \hat{b}_{0}^{+}|\mathrm{vac}\rangle, \\
& \left|\Psi_{n+1}\right\rangle=\hat{H}_{0}\left|\Psi_{n}\right\rangle-a_{n}\left|\Psi_{n}\right\rangle-b_{n}^{2}\left|\Psi_{n-1}\right\rangle,
\end{aligned}
$$

for $1 \leqslant n \leqslant L-1$, where

$$
\begin{aligned}
& a_{n}=\frac{\left\langle\Psi_{n}\left|\hat{H}_{0}\right| \Psi_{n}\right\rangle}{\left\langle\Psi_{n} \mid \Psi_{n}\right\rangle}, \\
& b_{n}^{2}=\frac{\left\langle\Psi_{n} \mid \Psi_{n}\right\rangle}{\left\langle\Psi_{n-1} \mid \Psi_{n-1}\right\rangle} .
\end{aligned}
$$


It is readily shown that $a_{n}=0(0 \leqslant n \leqslant L), b_{1}^{2}=2 t^{2}, b_{n}^{2}=t^{2}$ $(2 \leqslant n \leqslant L)$, and

$$
\left|\Psi_{n}\right\rangle=V(-t)^{n-1}\left[e^{i(n-1) \varphi} \hat{b}_{L-n+1}^{+}+e^{-i(n-1) \varphi} \hat{b}_{n-1}^{+}\right]|\mathrm{vac}\rangle .
$$

The algorithm automatically terminates after $n=L$, i.e., $\left|\Psi_{L+1}\right\rangle \equiv 0$.

After a proper normalization we find the $L+1$ basis states

$$
\begin{aligned}
& |d\rangle=\left|\Psi_{0}\right\rangle=\hat{d}^{+}|\mathrm{vac}\rangle, \\
& |0\rangle=\hat{C}_{0}^{+}|\mathrm{vac}\rangle,
\end{aligned}
$$

$$
\begin{aligned}
|n\rangle & =\sqrt{\frac{1}{2}}\left[e^{-i n \varphi} \hat{b}_{n}^{+}+e^{i n \varphi} \hat{b}_{L-n}^{+}\right]|\mathrm{vac}\rangle \\
& =\left\{\begin{array}{lll}
\hat{C}_{n}^{+}|\mathrm{vac}\rangle & \text { for } & 1 \leqslant n \leqslant(L-1) / 2, \\
i \hat{S}_{L-n}^{+}|\mathrm{vac}\rangle & \text { for } & (L+1) / 2 \leqslant n \leqslant L .
\end{array}\right.
\end{aligned}
$$

Up to a phase factor for the $S$ electrons, this is the same basis as used in the canonical transformation. The extra phase factor accounts for the electron transfer it between the $C$-electron and $S$-electron chains in Sec. II C.
[1] P. W. Anderson, Phys. Rev. 124, 41 (1961).

[2] P. W. Anderson, Rev. Mod. Phys. 50, 191 (1978).

[3] A. C. Hewson, The Kondo Problem to Heavy Fermions (Cambridge University Press, Cambridge, 1993).

[4] S. G. Jakobs, M. Pletyukhov, and H. Schoeller, Phys. Rev. B 81, 195109 (2010).

[5] M. Kinza, J. Ortloff, J. Bauer, and C. Honerkamp, Phys. Rev. B 87, 035111 (2013).

[6] J. F. Rentrop, V. Meden, and S. G. Jakobs, Phys. Rev. B 93, 195160 (2016).

[7] C. Zener, Phys. Rev. 81, 440 (1951).

[8] J. Kondo, Prog. Theor. Phys. 32, 37 (1964).

[9] R. Bulla, T. A. Costi, and T. Pruschke, Rev. Mod. Phys. 80, 395 (2008).

[10] D. E. Logan, M. P. Eastwood, and M. A. Tusch, J. Phys.: Condens. Matter 10, 2673 (1998).

[11] D. E. Logan and M. T. Glossop, J. Phys.: Condens. Matter 12, 985 (2000).

[12] M. R. Galpin, A. B. Gilbert, and D. E. Logan, J. Phys.: Condens. Matter 21, 375602 (2009).

[13] L. Borda, Phys. Rev. B 75, 041307(R) (2007).

[14] A. K. Mitchell, M. Becker, and R. Bulla, Phys. Rev. B 84, 115120 (2011).

[15] B. Lechtenberg and F. B. Anders, Phys. Rev. B 90, 045117 (2014)

[16] S. Florens and I. Snyman, Phys. Rev. B 92, 195106 (2015).

[17] S. Ghosh, P. Ribeiro, and M. Haque, J. Stat. Mech.: Theory Exp. (2014) P04011.

[18] A. Holzner, I. P. McCulloch, U. Schollwöck, J. von Delft, and F. Heidrich-Meisner, Phys. Rev. B 80, 205114 (2009).

[19] N. Andrei, Phys. Rev. Lett. 45, 379 (1980).

[20] P. B. Wiegmann, J. Phys. C 14, 1463 (1981).

[21] P. B. Wiegmann and A. M. Tsvelick, J. Phys. C 16, 2281 (1983).

[22] A. M. Tsvelick and P. B. Wiegmann, J. Phys. C 16, 2321 (1983).

[23] K. Schönhammer, Phys. Rev. B 42, 2591 (1990).
[24] F. Gebhard, Phys. Rev. B 41, 9452 (1990).

[25] C. A. Büsser, G. B. Martins, and A. E. Feiguin, Phys. Rev. B 88, 245113 (2013).

[26] A. Schwabe, M. Hänsel, M. Potthoff, and A. K. Mitchell, Phys. Rev. B 92, 155104 (2015).

[27] See Supplemental Material at http://link.aps.org/supplemental/ 10.1103/PhysRevB.99.165130 for the derivation of the results for the noninteracting SIAM (resonant-level model) and the Hartree-Fock theory, which includes Refs. [43-45].

[28] O. Legeza, F. Gebhard, and J. Rissler, Phys. Rev. B 74, 195112 (2006).

[29] A. E. Feiguin and C. A. Büsser, Phys. Rev. B 84, 115403 (2011).

[30] J. R. Schrieffer and P. A. Wolff, Phys. Rev. 149, 491 (1966).

[31] Z. M. M. Mahmoud and F. Gebhard, Ann. Phys. (Berlin) 527, 794 (2015).

[32] N. Kawakami and A. Okiji, Phys. Lett. A 86, 483 (1981).

[33] S. R. White, Phys. Rev. Lett. 69, 2863 (1992).

[34] S. R. White, Phys. Rev. B 48, 10345 (1993).

[35] O. Legeza, J. Röder, and B. A. Hess, Phys. Rev. B 67, 125114 (2003).

[36] O. Legeza and J. Sólyom, Phys. Rev. B 70, 205118 (2004).

[37] K. Yamada, Prog. Theor. Phys. 53, 970 (1975).

[38] We correct a typo in the expression in Ref. [21] by replacing $\pi^{1 / 2}$ with $\pi^{3 / 2}$

[39] S. Doniach and M. Šunjić, J. Phys. C 3, 285 (1970).

[40] A. Okiji and N. Kawakami, Solid State Commun. 43, 365 (1982).

[41] L. Fritz and M. Vojta, Rep. Prog. Phys. 76, 032501 (2013).

[42] T. Linneweber, J. Bünemann, Z. M. M. Mahmoud, and F. Gebhard, J. Phys.: Condens. Matter 29, 445603 (2017).

[43] Wolfram Research, Inc., Mathematica 10 (Champaign, IL, 2016).

[44] J. Goldstone, Proc. R. Soc. London A 239, 267 (1957).

[45] D. Ruhl and F. Gebhard, J. Stat. Mech.: Theory Exp. (2006) P03015. 